Chapman University

Chapman University Digital Commons

$5-17-2017$

BendableSound: An Elastic Multisensory Surface Using Touchbased interactions to Assist Children with Severe Autism During Music Therapy

Franceli L. Cibrian

Oscar Peña

Deysi Ortega

Monica Tentori

Follow this and additional works at: https://digitalcommons.chapman.edu/engineering_articles

Part of the Music Therapy Commons, Other Electrical and Computer Engineering Commons, Other Psychiatry and Psychology Commons, Pediatrics Commons, and the Special Education and Teaching Commons 


\section{BendableSound: An Elastic Multisensory Surface Using Touch-based interactions to Assist Children with Severe Autism During Music Therapy}

\section{Comments}

NOTICE: this is the author's version of a work that was accepted for publication in International Journal of Human-Computer Studies. Changes resulting from the publishing process, such as peer review, editing, corrections, structural formatting, and other quality control mechanisms may not be reflected in this document. Changes may have been made to this work since it was submitted for publication. A definitive version was subsequently published in International Journal of Human-Computer Studies, volume 107, in 2017. https://doi.org/10.1016/j.ijhcs.2017.05.003

The Creative Commons license below applies only to this version of the article.

\section{Creative Commons License}

\section{(c) $† \odot \ominus$}

This work is licensed under a Creative Commons Attribution-Noncommercial-No Derivative Works 4.0 License.

\section{Copyright}

Elsevier 


\title{
BendableSound: An Elastic Multisensory Surface using Touch-based interactions to Assist Children with Severe Autism during Music Therapy
}

Authors and affiliations: Franceli L. Cibrian, Oscar Pena, Deysi Ortega, Monica Tentori. \{franceli, opena, dortega\}@cicese.edu.mx, mtentori@cicese.mx. Computer Science Department, Center for Scientific Research and Higher Education of Ensenada, Mexico (CICESE)

Corresponding Author: Monica Tentori, mtentori@cicese.mx, Computer Science Department, Center for Scientific Research and Higher Education of Ensenada, Mexico (CICESE)

\begin{abstract}
Neurological Music Therapy uses live music to improve the sensorimotor regulation of children with severe autism. However, they often lack musical training and their impairments limit their interactions with musical instruments. In this paper, we present our co-design work that led to the BendableSound prototype: an elastic multisensory surface encouraging users to practice coordination movements when touching a fabric to play sounds. We present the results of a formative study conducted with 18 teachers showing BendableSound was perceived as "usable" and "attractive". Then, we present a deployment study with 24 children with severe autism showing BendableSound is "easy to use" and may potentially have therapeutic benefits regarding attention and motor development. We propose a set of design insights that could guide the design of natural user interfaces, particularly elastic multisensory surfaces. We close with a discussion and directions for future work.
\end{abstract}

Keywords: multisensory interfaces; elastic surfaces; neurologic music therapy; autism; strength and timing regulation. 


\section{Introduction}

Autism is a neurological disorder associated with impairments in attention, social interaction and behavior (American Psychiatric Association, 2013). Most children with severe autism exhibit significant motor coordination (Fournier et al., 2010; Green et al., 2009; Piochon et al., 2014; Torres and Donnellan, 2015) and sensorimotor impairments (Torres and Donnellan, 2015). As a consequence, children with severe autism may have limited motor control defaulting their ability to redirect appropriately their movements to respond to sensory stimuli (LaGasse and Hardy, 2013; Staples and Reid, 2010). For example, when children with severe autism move their body to respond to stimuli, their reaction time is usually off by either reacting later or in advance. Additionally, children with severe autism frequently use insufficient or excessive amount of strength (Fournier et al., 2010).

Neurological Music Therapy (NMT) is increasingly being used to support the sensorimotor regulation of individuals with autism with promising clinical results (LaGasse and Hardy, 2013). NMT is the therapeutic use of music to improve individual's cognitive, sensory, and motor dysfunctions due to a neurologic [disorder] of the human nervous system (Thaut and Hoemberg, 2014). Particularly, the Therapeutic Instrumental Music Performance (TIMP) is a technique used in in NMT where therapists play musical instruments to help patients practice physical exercises (Mertel, 2014). In a typical TIMP-NMT session, musical instruments, not being played in a traditional manner, are arranged in different positions to guide patients' movements (Mertel, 2014). For example, when working with two musical instruments, a therapist uses one musical instrument as the starting point of the movement and the second one as the ending point.

Current TIMP-NMT sessions rely heavily on the use of traditional musical instruments ${ }^{1}$. However, available traditional musical instruments might not be appropriately designed to support the needs of children with severe autism, presenting excessive cognitive load and demanding musical training (Magee, 2006). As a consequence, children with severe autism found it difficult to interact with traditional musical instruments, they often lose their attention, become frustrated (Burland and Magee, 2012), and abandon the therapy without meeting their goals. Designing tools to support the needs of children with severe autism during TIMP-NMT is not an easy task. These tools should be ease to use, attractive, and should use multi-sensory stimuli and enable a natural interaction. Particularly, the use of multisensory experiences could allow children with autism to maintain their attention and engagement during therapies (Cuvo et al., 2001). There is evidence that the use of adequate visual, auditory, and haptic stimuli leads to less caregiver assistance (Schaaf et al., 2014). As most interventions for children with autism (including some techniques used in music therapy) are more pragmatic and their main goal is to enable skills generalization; fading out prompts is paramount to enable children to apply the newly learned skills during therapy to real-life situations. The proper correspondence among each of the multisensory stimuli helps to process adequately and integrate sensory information (Schaaf et al., 2014).

It has been proved that interactive surfaces, any kind of surface augmented with multi-touch capabilities, offering a natural and casual interaction, and removing the complexity of the input interaction mechanism (Putnam and Chong, 2008); can support the interactions of individuals with

\footnotetext{
${ }^{1}$ We adopted the term traditional musical instrument to define instruments from both popular music and classical music. These instruments are designed for precise interaction, and usually require a high level of expertise from users to play them (Visi et al., 2014). Examples of traditional musical instruments used in NMT-TIMP include percussions, a guitar, a violin, a piano, or their electronic version (Magee, 2006; Mertel, 2014).
} 
disabilities with music (Boulay et al., 2011; Gorman et al., 2007; Hobbs and Worthington-Eyre, 2008; Oliveros et al., 2011). Interactive surfaces also provide the multisensory experience children with severe autism need to stay focused during therapeutic interventions (Villafuerte et al., 2012). Deformable displays allow users to use gestures and explore novel ways of interaction. Deformable surfaces enable users to vary the amount of pressure used when touching the surface by including an extra dimension (Müller et al., 2015). Deformable surfaces could be either flexible including those deformable displays that are highly flexible and may allow for permanent deformation; or elastic including elastic displays like fabrics allowing only for temporary deformation (Grunder et al., 2013).

Particularly, our research aims to understand the design space of elastic multisensory surfaces that, in contrast with traditional musical instruments, can integrate a huge variety of sounds that could provide guidance to users about their movements. Elastic multisensory surfaces may benefit children with severe autism as they provide a rich multisensory experience and enable them to understand strength variations (Troiano et al., 2014). We hypothesize that elastic multisensory surfaces are better tools than traditional musical instruments to support children with autism during TIMP-NMT sessions.

The main contributions of this work are ${ }^{2}$

- A prototype of an elastic multisensory surface, called BendableSound, developed to encourage children with autism to practice coordination movements while playing sounds in a natural way by using touch-based interactions in a multisensory environment.

- Qualitative and quantitative empirical evidence showing how elastic multisensory surfaces, augmented with multisensory feedback, are better tools than traditional musical instruments to help children with severe autism stay focused and uncover novel tactile experiences during TIMPNMT sessions.

- Reflections from our co-design process and a set of design insights that could guide the design of natural user interfaces, particularly elastic surfaces, to design multisensory experiences.

\section{Related Work}

In this section, we first describe research related to the design of ubiquitous technology using multisensory stimuli to support the control of movements of individuals with disabilities; some of them support music therapy interventions. We then describe research around elastic displays and deformable surfaces in general. We close with a discussion of other interactive surfaces facilitating users' interactions with live music.

\subsection{Multisensory environments to support individuals with disabilities}

Research has explored the design and development of interactive surfaces using visual stimuli to control the movements of individuals with autism (Parés et al., 2005; Ringland et al., 2014). For example, MEDIATE (Parés et al., 2005) is an interactive surface enabling children with autism to use their body to control the digital information displayed on the surface. A deployment study, with about

\footnotetext{
${ }^{2}$ This paper extends our previous results describing our iterative design process (Cibrian et al., 2016a) and an exploratory study conducted with toddlers to investigate the value of BendableSound for the early development in toddler classrooms (Cibrian et al., 2016b). In contrast, in this paper, we build upon our previous results and we present a new design of the BendableSound prototype, results from a formative study conducted to investigate the perception of psychologist-teachers to this new design, and results from a deployment study to investigate the impact of BendableSound in supporting the therapies of children with autism.
} 
90 individuals with autism using MEDIATE, showed that the visual interaction allows individuals to be creative and self-express within the environment. Other projects have demonstrated the usefulness of using sonification techniques to provide feedback to users about their movements during sports (Cesarini et al., 2016; Schaffert and Mattes, 2015), when dancing (Grosshauser et al., 2012), and for rehabilitation purposes (Rosati et al., 2013; Sigrist et al., 2014; Singh et al., 2015). For example, the use of sonification techniques helps individuals with chronic pain to use sounds as a feedback mechanism to guide their movements (Singh et al., 2015).

These projects show that a multisensory environment facilitates individuals with disabilities to interact with multisensory stimuli, but there is no evidence of how such solutions could support the sensorimotor regulation of children with severe autism and there is limited research in exploring novel haptic experiences with multiple sensory stimuli.

\subsection{Deformable interfaces}

Beyond exploring technologies that limit our senses of vision and audition, others have proposed the use of deformable materials to design novel haptic and tactile experiences. Flexible interfaces have been used to improve musical experiences of children with disabilities (Grierson and Kiefer, 2013) who experience more challenges than typical children when interacting with traditional musical instruments. For example, NoiseBear (Grierson and Kiefer, 2013) is a wireless malleable soft controller designed to help children with autism to create sounds and music through gestural interactions. A pilot study of the use of NoiseBear showed that children with severe autism found NoiseBear engaging when creating music, but lacking the visual stimuli to provide them with a complete multisensory experience.

On the other hand, elastic displays which, given their elasticity allow users to change the surface by pulling, pushing or twisting them (Müller et al., 2015; Sahoo et al., 2016; Troiano et al., 2014; Yun et al., 2013) have been proposed to support navigation (Yun et al., 2013), and manipulate physical (Müller et al., 2015) and multimedia (Cassinelli and Ishikawa, 2005) information. Users can either use one finger, their complete hand, or unimanual and bimanual gestures (Müller et al., 2015; Troiano et al., 2014). For example, DepthTouch (Müller et al., 2015) is an elastic display mimicking the gravitational forces between spheres. The spheres react according to the force applied when users push the elastic surface. Spheres can either repulse or attract according to the force of gravity. The use of DepthTouch shows the elastic display is easy to understand and help users to create quickly a mental model of how its interaction works (Müller et al., 2015).

These projects suggest that the affordances of elastic displays, including haptic sensations combined with visual stimuli, help users to understand quickly how to interact with the surface. However, to the best of our knowledge, there are no studies reporting results from evaluations of elastic multisensory displays to support children with autism. Descriptions of such real-life deployments are urgently needed to better understand their interaction experience and their potential therapeutic value for this population.

\subsection{Musical interfaces}

Previous research on interactive surfaces has explored innovative ways to help users learn how to play music through rhythm games (e.g., Guitar Hero, Rock Band) or by augmenting traditional musical instruments with interactive information (Rogers et al., 2014; Xiao et al., 2016). For example, Andantino (Xiao et al., 2016) is an interactive surface projecting animated figures on top of a piano. Animated figures represent a wide range of musical elements like sound quality, moods in the music or harmonies. The aim of Andantino is to provide an enjoyable music learning environment for 
children. These projects emphasize the importance of using multisensory stimuli to improve the overall experience of playing music. Other musical interfaces have explored the use of tangible interactions to support children when creating music (Ichino et al., 2014; Jordà et al., 2007). For example, Vuzik (Ichino et al., 2014) is an interactive display mimicking a stand-up easel enabling typical children to create music while painting on a canvas. A study with 14 children (aged 9 to 12 years old) found Vuzik was easy to use and provided better support for creativity than a software helping children to compose music running on a traditional desktop interface. Although some of these interactive surfaces are commercially available and used by expert musicians, they have not yet been evaluated with children with severe autism.

Other interactive interfaces have been proposed to support individuals with motor disabilities during therapeutic interventions (e.g., MINWii (Boulay et al., 2011), Music Maker (Gorman et al., 2007), Movement-to-Music (Hobbs and Worthington-Eyre, 2008)). For example, MINWii (Boulay et al., 2011) is a game for patients with dementia using music therapy techniques that helps individuals to play different songs. A deployment study of the use of MINWii shows that combining both tangible and gestural interactions could ease the process of playing music for patients with motor and cognitive impairments. However, MINWii does not provide an appropriate dynamic that could allow patients with motor impairments to practice how to self-regulate their strength and improve their movement timing.

Although, this body of work has made the compelling argument that multisensory environments are appropriate for children with autism (Parés et al., 2005; Ringland et al., 2014), and particularly deformable surfaces facilitate their interactions with live music (Grierson and Kiefer, 2013; Troiano et al., 2015); to our knowledge, there is no evidence of the design of a device devoted to support TIMP-NMT sessions for children with severe autism. Our design differs from others available in the literature as it combines multiple sensory stimuli and provides children with severe autism with challenges related to sensory integration and motor development using techniques from therapeutic interventions.

\section{Designing the BendableSound prototype}

We followed an iterative user-centered design methodology to design BendableSound, a large-scale elastic multisensory surface that allows users to make music when tapping and touching on top of the canvas (Figure 1-left). Users can play the sounds of different musical instruments. BendableSound displays a 3D background showing an animation of a nebula with translucent space-based elements, like stars and planets, appearing on top of the fabric. BendableSound is a system using a Kinect sensor, speakers, and an ultra-short throw projector placed behind a spandex fabric (Figure 1-right). The design of BendableSound involved three iterations. In total, we conducted 12 semi-structured

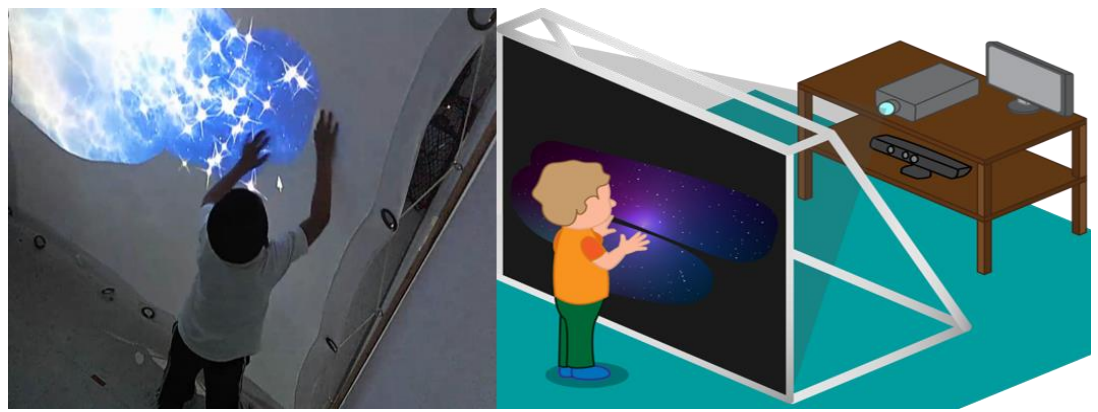

Figure 1. The BendableSound prototype. A child discovering sounds and the space nebula (left). A mock-up of BendableSound (right). interviews, 10 design sessions, and completed 21.5 hours of passive observation (Table 1). 
Table 1. Details of the data collected and activities conducted when designing BendableSound

\begin{tabular}{|c|c|c|c|c|c|}
\hline Iteration & Activity & Purpose & Outcome & \# of participants & Job description \\
\hline \multirow{6}{*}{ I } & $\begin{array}{l}4 \quad \text { Semi-structured } \\
\text { Interviews }\end{array}$ & $\begin{array}{l}\text { Identify the activities conducted, artifacts, and, technology used at } \\
\text { 'Pasitos' during current activities, the common problem children with } \\
\text { autism face during their day and the type of prompting, incentives, and } \\
\text { reinforcement used during class }\end{array}$ & \multirow{2}{*}{$\begin{array}{l}\text { An understanding of the everyday practices } \\
\text { and the problems children face during these } \\
\text { activities }\end{array}$} & 4 & $\begin{array}{l}1 \text { Psychologist-teachers (women) } \\
\text { from Pasitos } \\
2 \text { Musicians (Male) } \\
1 \text { Father of a child with autism }\end{array}$ \\
\hline & $\begin{array}{l}15 \text { hours of passive } \\
\text { Observation }\end{array}$ & $\begin{array}{l}\text { Observe the current activities and the common problems that children } \\
\text { face in the classes at Pasitos and playing in the playground }\end{array}$ & & 38 & $\begin{array}{l}8 \text { Psychologist-teachers } \\
30 \text { Children with autism }\end{array}$ \\
\hline & \multirow{4}{*}{4 Design sessions } & Define a set of preliminary low-fidelity prototypes & $\begin{array}{l}\text { A set of design scenarios and low-fidelity } \\
\text { prototypes }\end{array}$ & 2 & $2 \mathrm{HCI}$ experts \\
\hline & & $\begin{array}{l}\text { Discuss advantages and disadvantage of different prototypes of } \\
\text { interactive surface, and choose one from the alternatives }\end{array}$ & $\begin{array}{l}\text { Three low fidelity prototypes such as } \\
\text { interactive floor, tabletop and elastic } \\
\text { multisensory surface. }\end{array}$ & 6 & $\begin{array}{l}1 \text { Designer, } \\
1 \text { Interactive surface expert, } \\
3 \text { HCI, } \\
1 \text { Musician }\end{array}$ \\
\hline & & $\begin{array}{l}\text { Observe typical interactions of neurotypical children with different } \\
\text { prototypes, understand their preferences, and define what gestural } \\
\text { interactions are appropriate for the chosen prototype }\end{array}$ & $\begin{array}{l}\text { A set of movement that children could perform } \\
\text { on an elastic multisensory surface }\end{array}$ & 6 & $\begin{array}{l}2 \text { Girls, } \\
4 \text { HCI experts }\end{array}$ \\
\hline & & Discuss the animation of the interactive surface & $\begin{array}{l}\text { A visualization that could be integrated into an } \\
\text { elastic multisensory surface }\end{array}$ & 5 & $\begin{array}{l}\text { 1 Psychologist, } \\
4 \text { HCI experts }\end{array}$ \\
\hline \multirow{4}{*}{ II } & $\begin{array}{l}3 \text { Semi-structured } \\
\text { Interviews }\end{array}$ & $\begin{array}{l}\text { Identify the physical therapy lifecycle, including activities conducted, } \\
\text { artifacts and strategies for clinical case management and assessment, and } \\
\text { the common motor problems children with autism face. }\end{array}$ & $\begin{array}{l}\text { An understanding of physical therapy and } \\
\text { motor development techniques used during the } \\
\text { motor control exercise }\end{array}$ & 3 & $\begin{array}{l}1 \text { Physical therapist (male) } \\
1 \text { Psychologist (women) } \\
1 \text { Neuropsychologist (women) }\end{array}$ \\
\hline & $\begin{array}{l}\text { 0.5 hours of passive } \\
\text { Observation }\end{array}$ & Observe the interactions of children with autism using BendableSound. & $\begin{array}{l}\text { Initial reactions of children with autism to } \\
\text { BendableSound }\end{array}$ & 8 & $\begin{array}{l}7 \text { Children with autism using } \\
\text { BendableSound } \\
1 \text { Psychologist }\end{array}$ \\
\hline & \multirow{2}{*}{3 Design sessions } & $\begin{array}{l}\text { Discuss the appropriate movements that children could perform in an } \\
\text { elastic multisensory surface }\end{array}$ & $\begin{array}{l}\text { A set of movement that children with autism } \\
\text { perform using BendableSound }\end{array}$ & 4 & $\begin{array}{l}1 \text { Physiotherapy } \\
3 \text { HCI experts }\end{array}$ \\
\hline & & $\begin{array}{l}\text { Define the sequences of movements more appropriate to strength and } \\
\text { timing regulation }\end{array}$ & $\begin{array}{l}\text { The sequences of movements more appropriate } \\
\text { to strength and timing regulation }\end{array}$ & 3 & $\begin{array}{l}1 \text { Neuropsychologist } \\
2 \mathrm{HCI} \text { experts }\end{array}$ \\
\hline \multirow{7}{*}{ III } & $\begin{array}{l}5 \text { Semi-structured } \\
\text { Interviews }\end{array}$ & $\begin{array}{l}\text { Identify the activities conducted, artifacts, and technology used during } \\
\text { music-therapy session and music activities, and the type of prompting, } \\
\text { incentives and reinforcement used during music therapy session and } \\
\text { music based activities }\end{array}$ & \multirow{4}{*}{$\begin{array}{l}\text { Understanding of a music therapy lifecycle and } \\
\text { techniques using in neurologic music therapy } \\
\text { session }\end{array}$} & 4 & $\begin{array}{l}2 \text { Music therapist. (Female and male) } \\
2 \text { Music teacher (Female and male) }\end{array}$ \\
\hline & \multirow{3}{*}{$\begin{array}{l}6 \text { hours of passive } \\
\text { Observation }\end{array}$} & Observer eight music classes of children with autism attending to Pasitos & & 10 & $\begin{array}{l}8 \text { Children with autism } \\
2 \text { Music teacher }\end{array}$ \\
\hline & & $\begin{array}{l}\text { Observe two Music therapy sessions of children with autism attending to } \\
\text { Pasitos }\end{array}$ & & 3 & $\begin{array}{l}2 \text { Children with autism } \\
1 \text { Music therapist }\end{array}$ \\
\hline & & Observe two Dance classes of two groups of children at Pasitos. & & 14 & $\begin{array}{l}10 \text { Children with autism } \\
2 \text { Dance teacher } \\
2 \text { Psychologist-teachers }\end{array}$ \\
\hline & \multirow{3}{*}{3 Design sessions } & Define the musical elements arrangement in BendableSound & $\begin{array}{l}\text { The match between the movement and the } \\
\text { musical elements }\end{array}$ & 4 & $\begin{array}{l}2 \text { Music-teacher } \\
2 \text { HCI experts }\end{array}$ \\
\hline & & $\begin{array}{l}\text { Discuss the agreement between the visual and auditory stimuli with the } \\
\text { haptic experience }\end{array}$ & $\begin{array}{l}\text { The match between the movements, musical } \\
\text { elements and visualization }\end{array}$ & 5 & $\begin{array}{l}1 \text { Music therapist } \\
4 \text { HCI experts }\end{array}$ \\
\hline & & $\begin{array}{l}\text { Define the levels of BendableSound integrating all the stimuli, sequences } \\
\text { of movement and TIMP Neuro-MT elements. }\end{array}$ & The dynamic's game of BendableSound & 7 & $\begin{array}{l}1 \text { Multimedia designer } \\
1 \text { psychologist } \\
5 \text { HCI experts }\end{array}$ \\
\hline
\end{tabular}




\subsection{Data collection}

For each iteration, we first conducted semi-structured interviews with specialists to understand the potential context of the use of BendableSound and observed children with severe autism in different activities at "Pasitos" 3 (Table 1). In order to have participants actively involved in the design process, we supplement our interviews and observations with design sessions with specialists (Table 1). During design sessions, all the participants were considered to be equal partners in the design process, and one HCI expert acted as the moderator of the sessions. Specialists were encouraged to brainstorm and reflect on design ideas, propose low-fidelity prototypes and discuss advantages and disadvantages of each prototype (Wendell et al., 2004). At the end, specialists were asked to choose one prototype from all the alternatives they have proposed during the design sessions.

\subsection{Data analysis}

For data analysis, we transcribed all the interview's data and observation reports. Data analysis included the use of qualitative techniques, such as, open and axial coding (Strauss \& Corbin, 1998) to score our interviews and then group our codes in an affinity diagram. To analyze these data, we used the software atlas.ti ${ }^{4}$. All the data collected during our design sessions was analyzed using techniques from rapid contextual design (Wendell et al., 2004) and were materialized as sketches, storyboards and new ideas for potential activities to incorporate on the proposed prototypes.

\subsection{Findings}

In this subsection, we describe the main findings of each iteration.

\subsubsection{Iteration 1: Defining the gameplay and the visual stimuli}

Our qualitative results indicate that an elastic multisensory surface was the preferable prototype due to its novel way of interaction and potential innovative interaction experience. Specialists suggested including concepts commonly used during early development instruction, like real-life environments as potential visual themes for the prototype. Specialists selected the space theme as the most appropriate visualization for this population; explaining that it is gender neutral, sturdy and age appropriate. Specialists proposed a set of open-ended activities mimicking the improvisation activities conducted during a TIMP-NMT session and some goal-oriented tasks imitating the "companion role" of therapists. These results are consistent with others reported in the literature emphasizing that activities that might enable the long-term engagement of children should involve the making of music in a free manner (Srinivasan and Bhat, 2013) and should promote the integration of both simple and complex activities (Darrow, 2009).

To combine the improvisation activities with goal-oriented tasks conducted during a TIMP-NMT session, specialists suggested to include activities similar to the activity of drawing. Interactive surfaces proposed in the literature have shown the drawing activity, following an open-ended interaction model, is appropriate for children with autism (Hourcade et al., 2012; Parés et al., 2005; Ringland et al., 2014). For the goal-oriented task, specialists suggested the use of avatars and visual

\footnotetext{
3 "Pasitos" www.pasitos.org is a school-clinic located in Tijuana, Mexico where 18 psychologists-teachers attend to close to 60 children with severe and mild autism. Both our formative and summative studies for the development of BendableSound and its evaluation were conducted at this clinic.

${ }^{4}$ http://atlasti.com, a software to support qualitative research.
} 
stimuli to provide step-by-step guidance for children with autism. Both, avatars and visual supports are commonly used when designing goal-oriented activities for children with autism and are instrumental in giving them the structure they need during therapies (Hayes et al., 2010; Hopkins et al., 2011; Tartaro and Cassell, 2008). Specialists also proposed to use the piano as it is one instrument typically used during TIMP-NMT (Magee, 2006; Wigram Tony, Pedersen Inge Nygaard, 2011). Particularly, in Pasitos the piano is being used during music lessons.

These findings guide the design and develop the first version of the BendableSound prototype (Ortega et al., 2015). This first version of BendableSound supports two open-ended activities and one goaloriented task. In the first activity, children must erase a black layer covering the animation of space nebulas (Figure 2-left). In the second activity, children move space-based elements randomly appearing on top of the fabric canvas that will reproduce sounds when touched or moved (Figure 2right). In the third activity, an astronaut appears providing children with guidance on how to play a nursery rhyme by touching a blinking star (Ortega et al., 2015).

However, specialists expressed this version of BendableSound does not adequately mimic motor therapeutic interventions, as it does not provide feedback to children about what movements they need to conduct. Thus, we conducted a second iteration to uncover the appropriate movements to incorporate into our prototype.
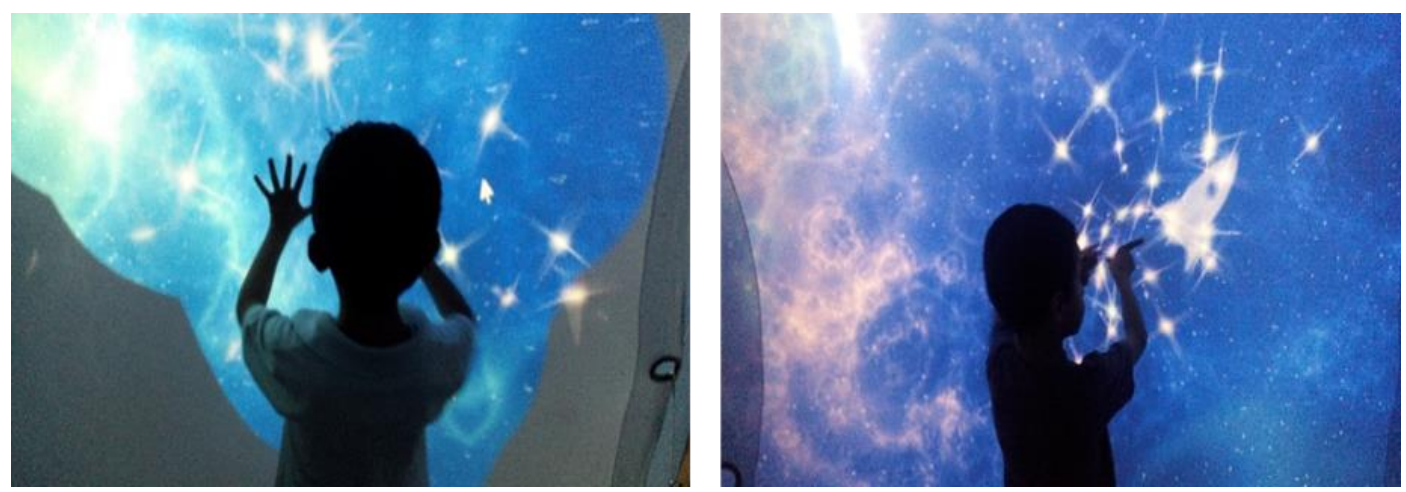

Figure 2 The BendableSound preliminary prototype. A participant playing the first activity of BendableSound erasing the black smog covering the space nebula by tapping throughout the fabric (left). A participant playing the second activity moving a spaceship to make piano sounds (right).

\subsubsection{Iteration 2: Designing the haptic experience}

The movements, that specialists selected, match with those typical movements used during motor therapeutic interventions for children with autism, especially those focused on improving movement timing and the self-regulation of their strength (Keele et al., 1987; Lundy-Ekman et al., 1991; Schmidt and Wrisberg, 2008). Specialists agreed that it is important to focus on one movement at the time and they selected a set of exercises that challenge children with severe autism at different levels. Specialists proposed an "exercise routine" demanding children to first push the fabric using their left hand, then the right hand, and finally alternating hands. In this manner, children are being challenged with movement patterns that demand from them practice bimanual coordination exercises (Brakke et al., 2007; Fagard and Wolff, 1991). Specialists also suggested adjusting the amount of strength being used when conducting the "exercise routine". They proposed activities that demand from children to either use extreme or little exertion of force at different levels of speed. This level of scaffolding during motor therapeutic interventions including strength and speed variations, and challenges around bimanual coordination has shown promising clinical results (Brakke et al., 2007; Fagard and Wolff, 1991). 
Specialists suggested changing the goal of the game by demanding children to use the appropriate amount of strength to help the rocket safely land on a planet. The rocket will appear on the left side or on right side of the fabric according to the hand children must use to push the rocket (See figures in Table 2), and throughout the game, children will be challenged with strength variations (Cibrian et al., 2016a). Specialists particularly proposed to include the following two activities:

- First, children must push the rocket to catapult its flight towards the next planet (Figure 3-left). The flight distance is determined by the amount of strength children use when pushing the fabric. The aim of this activity is to teach children how to follow bimanual coordination patterns (Brakke et al., 2007).

- Second, the amount of strength children use when pushing the fabric should catapult the rocket's flight that will collect an array of space-based elements that are either close or far from the rocket (Figure 3-center and right). Children must use enough strength to catch all the space-based elements, but not too much to skip them. This activity allows children to practice strength variations that could have a therapeutic benefit concerning force control (Keele et al., 1987; Lundy-Ekman et al., 1991; Schmidt and Wrisberg, 2008).

For both activities, children must complete a number of repetitions specified by the therapist. One rocket flight will count as one repetition.

Table 2. Set of movements proposed during participatory design sessions and included in BendableSound

\begin{tabular}{|c|c|c|c|c|}
\hline $\begin{array}{l}\text { Motor coordination } \\
\text { exercise }\end{array}$ & Movement & $\begin{array}{l}\text { Strength } \\
\text { variation }\end{array}$ & Timing Variation & Example \\
\hline $\begin{array}{l}\text { Uni-manual directed } \\
\text { movement }\end{array}$ & Push left-hand & $\begin{array}{l}\text { Free } \\
\text { Soft } \\
\text { Strong }\end{array}$ & $\begin{array}{l}\text { Free } \\
\text { Slow Rhythm } \\
\text { Fast Rhythm }\end{array}$ & \\
\hline $\begin{array}{l}\text { Uni-manual directed } \\
\text { movement }\end{array}$ & Push right-hand & $\begin{array}{l}\text { Free } \\
\text { Soft } \\
\text { Strong }\end{array}$ & $\begin{array}{l}\text { Free } \\
\text { Slow Rhythm } \\
\text { Fast Rhythm }\end{array}$ & \\
\hline $\begin{array}{l}\text { Bimanual } \\
\text { coordination } \\
\text { asynchronized }\end{array}$ & Push alternating & $\begin{array}{l}\text { Free } \\
\text { Soft } \\
\text { Strong }\end{array}$ & $\begin{array}{l}\text { Free } \\
\text { Slow Rhythm } \\
\text { Fast Rhythm }\end{array}$ & \\
\hline
\end{tabular}


Although these two activities better reflect the goal of the therapy, specialists explained that children needed more guidance. Thus, they suggested supplementing the visual and the haptic stimuli with appropriate sounds and music. This is consistent with previous findings in the literature regarding the benefits of using music and sounds to provide appropriate movement guidance (Keele et al., 1987; Lundy-Ekman et al., 1991; Schmidt and Wrisberg, 2008). Therefore, we conducted another iteration to design the appropriate auditory experience.
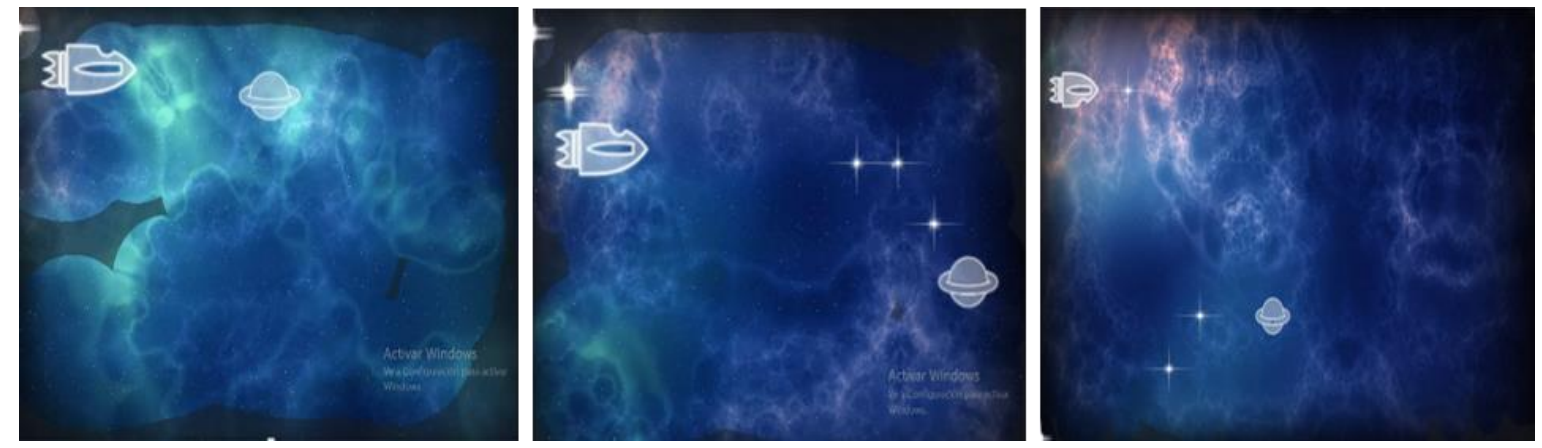

Figure 3. Screenshots of the activities available in BendableSound. Freely interaction to discover the black nebula (left). A rocket showing where to push to collect a note with and without base-pulse (center). A rocket showing where to push strong to collect a set of notes that are far away (bottom-right). A rocket showing where to push soft to collect a set of notes that are near (right).

\subsubsection{Iteration 3: Defining the auditory experience}

Specialists agreed that BendableSound should control the musical elements based on users' interactions. Musical elements could emphasize motor patterns and engage children in the therapeutic process (Srinivasan and Bhat, 2013; Thaut and Hoemberg, 2014). Musical elements should be organized as follows (Figure 4):

- For pitch, specialists agreed that sounds should be arranged from lower to higher in the vertical axis. This pitch arrangement could give children a sense of movement direction (Berger, 2002; Salgado-Montejo et al., 2016), given that, the most robust crossmodal correspondence of ascending pitch is elevation (Spence, 2011). For example, when the pitch goes up, the movement should go up as well. Specialists suggested that the notes selected should be from a song according to each child's preferences. The notes arrangement will determine the vertical position of the rocket. Children will use their strength to catapult the rocket and catch one or more musical notes represented by space-based elements. When catching the element children must hear the note or the array of notes being caught.

- For musical dynamic (intensity), specialists decided to vary the sound's volume according to the amount of strength being used to push the fabric. For example, a gentler push will decrease the volume. Dynamic should be akin to the energy level of music (Berger, 2002), to provide crossmodal correspondence between the sound and the amount of strength being used to push the fabric (Spence, 2011).

- For rhythm, specialists decided to control the music tempo using a "music pulse" being played in the background. This music pulse signals users when the music speeds up or slows down, enabling children to play the song according to the music tempo. However, children are not penalized when missing a beat. A "music pulse" being played in the background could improve the motor timing in children with autism (Hardy and Lagasse, 2013; Repp and Su, 2013), as children may potentially control their motor timing ability (Keele et al., 1987; Lundy-Ekman et al., 1991; Schmidt and Wrisberg, 2008).

- For timbre, specialists agreed that therapists must personalize the musical instruments according to each child's preferences. Musical instruments selected by specialists included a variety of 
musical instruments commonly available in music synthesizers. As timbre is one of the factors that is more problematic for people with sensory integration deficits (Berger, 2002), then, the personalization is highly recommended.

After our design team reviewed and agreed to all the modifications suggested to our prototype, we proceed with its development.

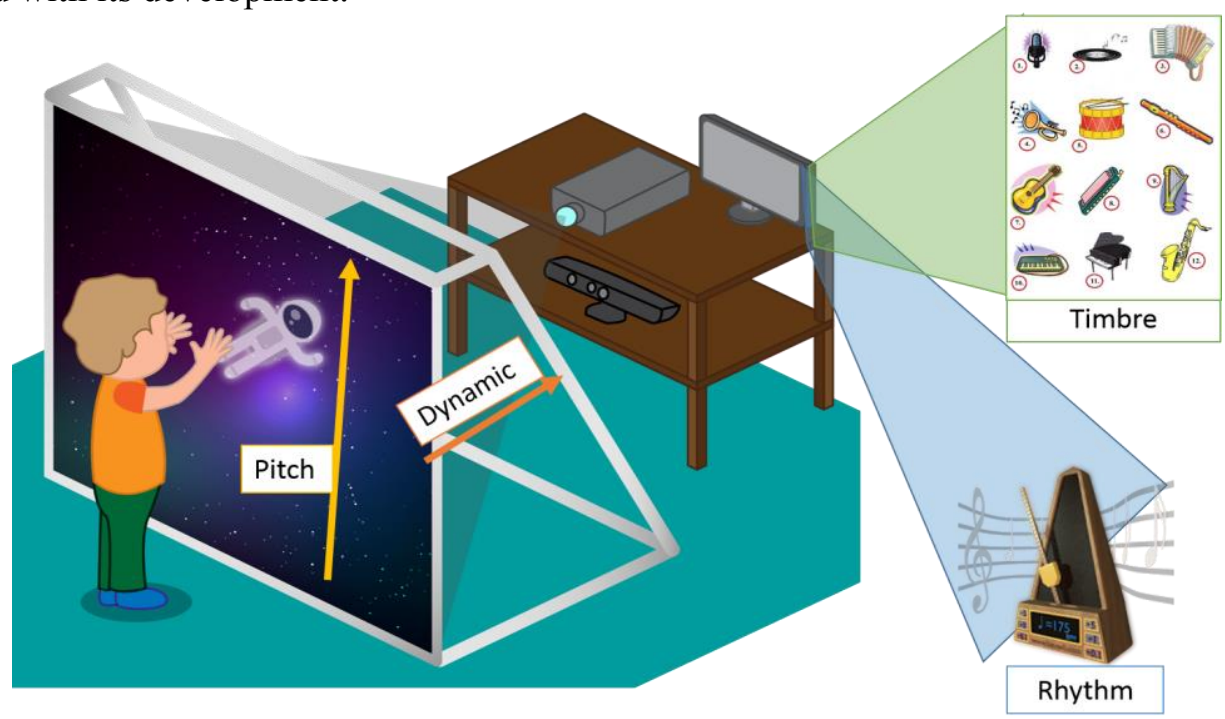

Figure 4. The musical elements arrangement in BendableSound: the pitch is represented by the musical notes arranged from lower to higher; the sound dynamic is represented with the volume and will vary according to the amount of strength; timbre is represented with the musical instruments sounds; and for rhythm, we use a music pulse as a background sound.

\section{Developing the BendableSound prototype}

The goal of the final version of BendableSound is that children use their strength to catapult the flight of a rocket to help it to land on a planet. During its journey, the rocket will collect notes of a nursery rhyme previously selected by the therapist. The BendableSound prototype works as follows:

First, children must interact in a free manner by erasing a black layer covering the space nebula (Figure 2-left). Second, a rocket appears on the left side of the fabric and children must tap on it to catapult its flight. While flying the rocket will collect one note of the nursery rhyme. Children must complete a certain amount of repetitions using their left hand. Then, the rocket moves to the right and children must complete the same number of repetitions using their right hand. Finally, children must alternate their hands and the rocket moves from left to right when appropriate (Figure 3-left). Third, children must use more strength to help the rocket reach the farthest planet and collect a set of notes (Figure 3-center). Then, they must push the rocket a little bit gentler to reach the closest planet (Figure 3-right). As in the past activity, children first use their left hand, then their right hand and finally they alternate their hands. Fourth, a music pulse appears and children must tap on the rocket according to music tempo to play the song (Figure 3-left). Finally, children go back to the first activity to play with the fabric in a free manner (Figure 2-left).

Therapists use a dedicated interface to select the musical instrument and the nursery rhyme according to each child's preferences. We added keyboard shortcuts that therapists could use to select a specific activity available in BendableSound or adjust the movement speed of the music. For selecting activities, teachers use numbers and the up/down arrows to speed up or slow down the music pulse.

The following scenario exemplifies how children with severe autism and their therapist could use BendableSound in practice. 
Marley is a 6-year-old child with severe autism who exhibits motor problems. Marley has difficulties when controlling his strength. Today, Marley is going to play BendableSound. The teacher Ms. Becky selects the piano instrument and the "Twinkle, twinkle little star" song. Marley pushes and swipes throughout the fabric to erase the "black layer" covering the space nebula. When Marley discovers the space nebula, a spaceship appears and Ms. Becky prompts Marley: "Marley, please push as hard as you can the fabric with your left hand". Marley touches the fabric softly and the rocket slowly flights collecting only one blinking star and one note of the song. Then, the rocket appears on the same side again and Marley pushes harder, and the spaceship collects four stars. Marley hears the collected notes and discovers the song "Twinkle, twinkle little star". Marley laughs. Ms. Becky congratulates Marley saying "Good job! Marley" and activates the last activity enabling Marley to play in a free manner with the music compasses and notes he discovered when playing with BendableSound.

The final version of BendableSound was developed using the Processing language, a 3D shader and TSPS library. We use the JavaSound API for controlling audio playback and a MIDI synthesizer.

\section{Evaluation methods}

We conducted two studies at Pasitos. The studies aim to answer the following questions:

- Do teachers perceive the design of BendableSound attractive and usable for children with severe autism?

- What is the overall interaction experience of children with severe autism with a multisensory deformable surface?

- What are the potential therapeutic benefits that BendableSound offers over a keyboard piano commonly used during TIMP-NMT sessions for children with severe autism?

\subsection{Formative study}

To answer the first two questions, we first conducted a formative survey-study to understand teachers' perception towards the design of BendableSound and discuss potential improvements to the design of BendableSound.

\subsubsection{Participants}

We recruited 18 teachers working at Pasitos. Although they are not the final users, they are the primary caregivers of these children at the clinic and were used as "proxies" to gather their perceptions. This "proxy" technique is commonly used when working with non-verbal populations (Tang and McCorkle, 2002).

\subsubsection{Procedure}

First, teachers used the first activity of BendableSound to get a better sense of its interaction experience. After that, we showcased all the activities of BendableSound and finally, we asked them to fill two questionnaires. First, teachers completed the System Usability Scale (SUS) (Brooke, 2013) survey using a 5-point Likert scale to measure user satisfaction. Then, they completed the User Experience Questionnaire (UEQ) (Laugwitz et al., 2008) using a 7-point Likert scale with bipolar items. We selected the SUS questionnaire as it is generic, quick, easy to administer and provides a

${ }^{5}$ A practice commonly used when working with non-verbal populations 
high-level usability perceptions from users. We selected the UEQ questionnaire as it enables a quick assessment of the user experience and has been released in Spanish (Rauschenberger et al., 2013). These surveys also had been widely used to evaluate usability (Bangor et al., 2008; Meldrum et al., 2012; Schonauer et al., 2011) and user experience (Nawaz et al., 2015; Santoso et al., 2014) of interactive interfaces. We also decided to use these questionnaires as both provide a benchmark of data that enable us to compare our results with those available in the literature. We analyzed the results from each questionnaire separately using the formulas specified by each survey (Brooke, 2013; Laugwitz et al., 2008).

\subsection{Deployment study}

To answer our last two questions, we conducted a deployment study at Pasitos.

\subsubsection{Participants}

Twenty-four children with severe autism ${ }^{6}$, between three to eleven years old, but with the same developmental age, were voluntarily enrolled in the study (Avg. age $=6.6$; $\mathrm{SD}=2.17$ ). Most of the participants were non-verbal, had attention problems, presented multiple sensory and cognitive impairments, and were not following a pharmacological treatment. None of the participants in the study had taken music lessons and had neither previously interacted with the piano nor with BendableSound. So, both conditions were novel experiences to the participants. We hired one psychologist trained in music therapy techniques who conducted the therapy sessions.

\subsubsection{Setting up and installation}

We equipped two therapy rooms at Pasitos with two video-cameras to monitor user's interactions, reactions, and movements. In the first room, we deployed BendableSound (Figure 5-left). In the second room, we removed all the potential available stimuli, like furniture or visual supports, and placed a keyboard piano on top of a table (Figure 5-right).
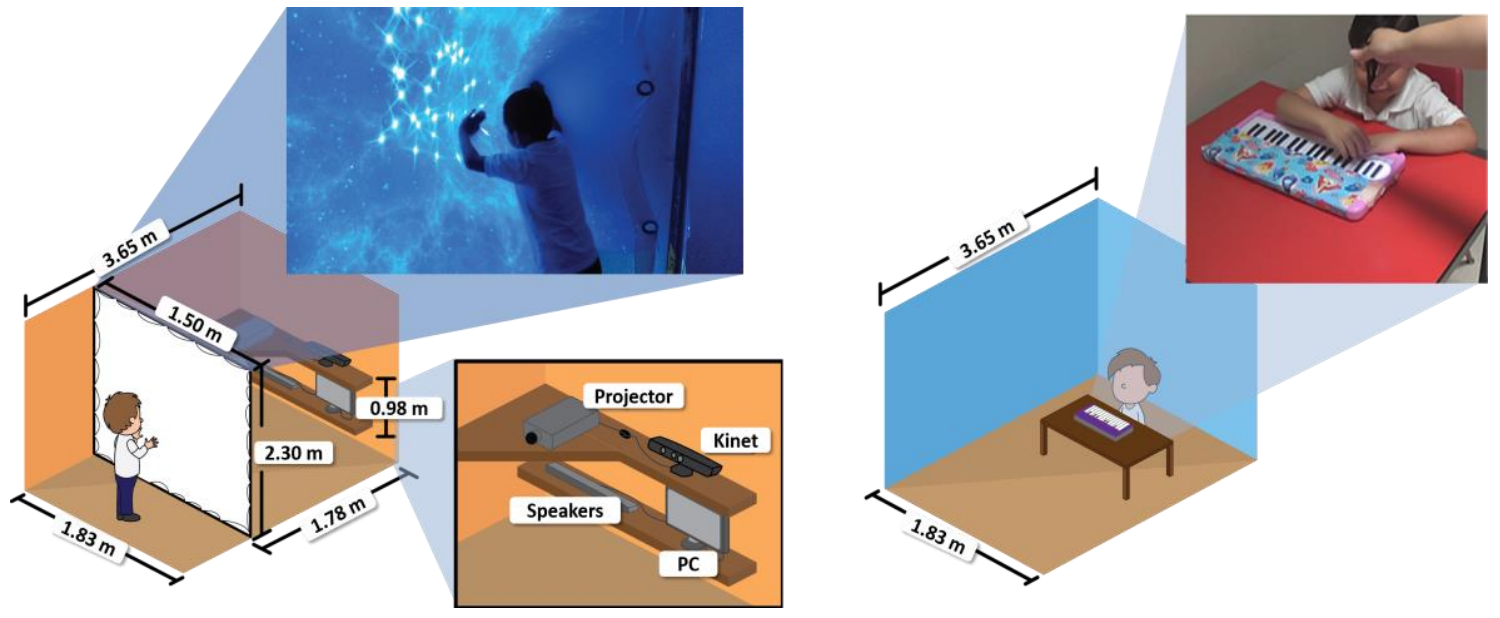

Figure 5. The installation of the two conditions. A mockup representation of the BendableSound room showing the hardware and software installation of BendableSound (left). A mockup installation of a traditional TIMP-NMT showing the installation of the keyboard piano and the laser light (right)

${ }^{6}$ For simplicity of reading, we will now refer to the children with severe autism participating in our evaluation study as participants. 
International Journal of Human-Computer Studies: Special Issue on "Multisensory HumanComputer Interaction" 2016

\subsubsection{Procedure}

All parents consented to the study on behalf of their children. The psychologist received a 15-minutes training to learn how to use BendableSound. Then, we conducted a 6-days within-subjects study. Participants attended a 5-minute music-therapy session, randomly rotating through the following two conditions:

- Using the first activity of BendableSound: participants interacted in a free manner for 5 minutes with BendableSound removing the black layer to discover the space nebula.

- Using a piano keyboard: participants freely interacted for 5 minutes with a keyboard piano commonly used during TIMP-NMT sessions at Pasitos

All sessions were video recorded, and logs were stored indicating participants' interactions with the fabric, for a total of 4 hours ( 2 hours per condition). Every day, upon completion of the experimental portion of the study, the psychologist was briefly interviewed (Total time $=53.9 \mathrm{~min}$; Avg. $=8.91$ min). These short and semi-structured interviews allowed us to follow up on anything that had happened during the session and included questions to uncover the differences between using the first activity of BendableSound and the keyboard piano.

Data analysis followed a mixed-method approach. To analyze video recordings, we used techniques inspired by structured observation (Mintzberg, 1970). Three researchers went through all the videos and systematically scored participants' behaviors following a pre-defined coding scheme (Table 3); The Inter-observer agreement, among these three researchers, was acceptable (Fleiss kappa Avg. $\kappa=$ 0.88). We estimated, for each participant and under each condition, the total and descriptive statistics of the time and frequency participants spend executing each behavior. Then, as our data distribution was non-parametric, we used the Wilcoxon Signed Rank-test to compare each behavior.

All interviews with the psychologist were recorded, transcribed and analyzed together. We used deductive analytical approaches, based on our initial questions focused on understanding the overall interaction experience of BendableSound. We used deductive coding to examine how observed behaviors and reported perceptions supported or contradicted our research questions. We additionally used inductive approaches to allow new themes to emerge from our data. To support our inductive analysis, we used open and axial coding, and affinity diagramming (Charmaz, 2006). Finally, we examined the themes regarding our inductive analysis to explain the results of our deductive analysis. Only one researcher coded the data, grouping the interview quotes that uncovered potential emerging themes. To analyze data, the researcher used the software atlas.ti.

Table 3. Brief definition of the codification scheme used to code the target behaviors

\begin{tabular}{|c|c|c|c|c|}
\hline Category & \multicolumn{2}{|c|}{ Target behavior } & Definition & Type of \\
\hline \multirow{5}{*}{$\begin{array}{l}\text { Users' } \\
\text { Behaviors }\end{array}$} & \multirow[t]{2}{*}{ Attention } & On task & The child is engaged and focused on the activity & Time \\
\hline & & Off task & The child is distracted & Time \\
\hline & \multirow[t]{3}{*}{ Emotions } & Negative & The child is crying (sad), frowning (angry), and screaming (fear). & Time \\
\hline & & Positive & The child is smiling (joy), clapping (happy), laughing (amused), & Time \\
\hline & & None & The child does not express any emotion & Time \\
\hline \multirow{7}{*}{$\begin{array}{l}\text { Users' } \\
\text { movements }\end{array}$} & \multirow{3}{*}{ Hand } & Finger & The children use one or more fingers to push the fabric & Frequency \\
\hline & & Palm & The children use one or both hands palms to push the fabric & Frequency \\
\hline & & Clenched fists & The children use one or both clenched fists to push the fabric & Frequency \\
\hline & Feet & Kick & The children kick the fabric with their foot & Frequency \\
\hline & \multirow[t]{2}{*}{ Head } & Immerse & The children use the whole head to push the fabric & Frequency \\
\hline & & Push with the face & The children use their face to push the fabric & Frequency \\
\hline & Back & Slip down & The children slip down backward over the fabric. & Frequency \\
\hline
\end{tabular}


International Journal of Human-Computer Studies: Special Issue on "Multisensory HumanComputer Interaction" 2016

\section{Results}

Overall, our results indicate teachers found the design of BendableSound to be "usable" and "attractive" and that both teachers and students found it "easy to use". Our results also show BendableSound might have potential therapeutic benefits regarding attention and motor development.

\subsection{Teachers' satisfaction of the design of BendableSound}

According to the SUS questionnaire (Brooke, 2013), on average, teachers agreed that the design of BendableSound was "acceptable" (Avg. $=72.63^{7}$, Figure 6), also, $83 \%$ of teachers rated it as above average (Brooke, 2013). This result suggests that the usability of BendableSound is above average compared to a benchmark of data from other studies using the SUS survey (Brooke, 2013). We found that $50 \%$ of teachers are satisfied with the design of BendableSound rating it as "good" and $11.1 \%$ rated it as "excellent". All the teachers found the new design of BendableSound is "easy to use". These results show that our new design of BendableSound is "good" and "usable".

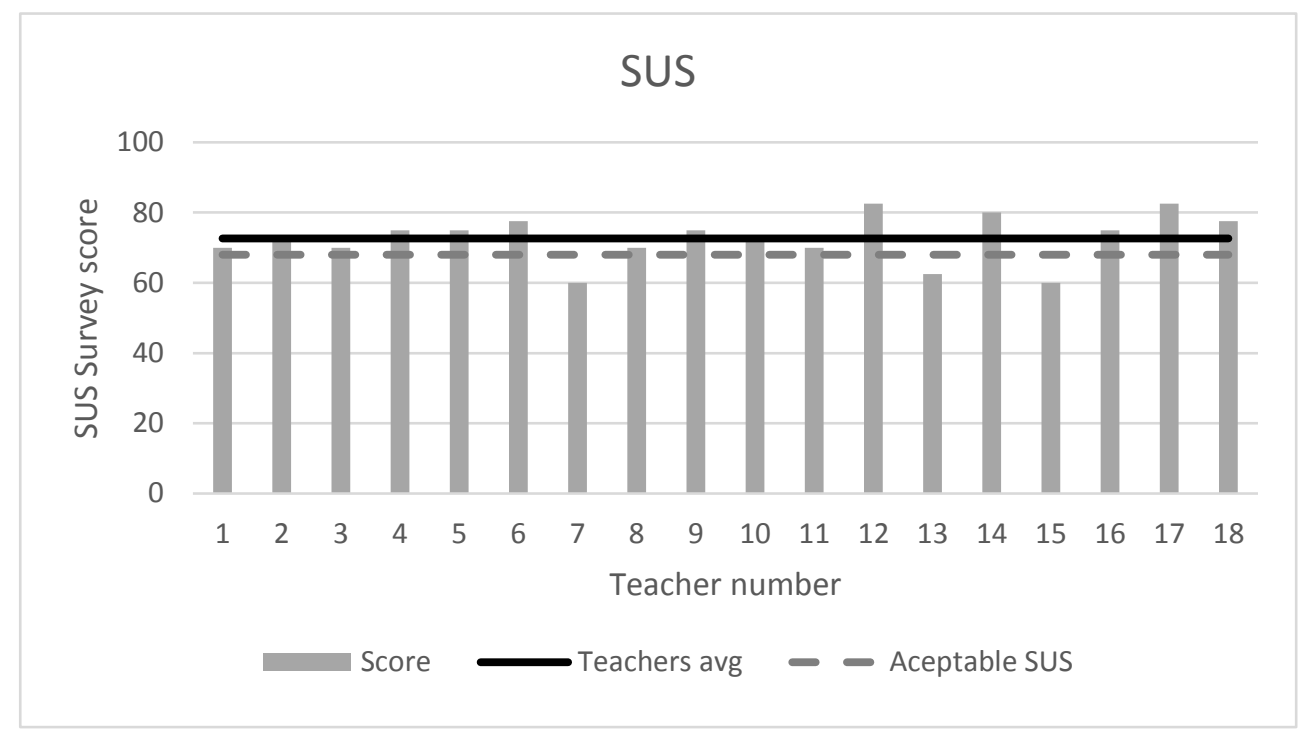

Figure 6. System usability scale (SUS) score per teacher of the design of BendableSound (Scores above 68 signifies "acceptable").

\subsection{Overall user experience}

Overall, our results show BendableSound has the potential to provide a more natural interaction and an intuitive multisensory experience. All teachers found BendableSound "attractive", "novel" and with an appropriate "learnability". Our results from our deployment study suggest elastic multisensory surfaces promote the discovery of new haptic experiences, and children with severe autism found BendableSound "easy to use", "engaging" and "novel".

\subsubsection{Teacher's perceptions of the user experience of BendableSound}

According to the UEQ survey (Rauschenberger et al., 2013), teachers were very satisfied with the new design of BendableSound as it meets their expectations (see Table 4 for more details of the

${ }^{7}$ The result of the questionnaire is a value between 1 and 100, where 1 signifies that a user found a system absolutely useless and 100 that a user found a system optimally useful. Results above 68 signify "acceptable".

${ }^{8}$ The ability of a user to easily get familiar with the product. (Rauschenberger et al., 2013). 
measurements and their score). Analyzing our results by theme (see the scales in Table 4), we found out teachers scored attractiveness very high (Avg. $=2.25$ out 3 ) and the categories related to novelty, dependability, and learnability were scored as high (Avg. above 1.5). These results could be partially explained because having multisensory environments in clinics for children with autism in Mexico is very expensive and most clinics lack such support. Thus, BendableSound was perceived highly novel. In contrast, efficiency was scored below average. This might mean that teachers could feel a little intimidated to use music during their everyday practices as they lack musical training.

Table 4. Teachers responses to the UEQ questionnaire to measure BendableSound design ( $n=18$; means are scaled from -3 to +3 . Thus, 3 represents the most negative answer, 0 a neutral answer, and +3 the most positive answer).

\begin{tabular}{|l|l|l|l|l|}
\hline \multicolumn{5}{|c|}{ Confidence intervals (p=0.05) per scale } \\
\hline Scale & Mean & Std. Dev. & \multicolumn{2}{l|}{ Confidence interval } \\
\hline Attractiveness & 2.25 & 0.77 & 1.89 & 2.61 \\
\hline Learnability & 1.85 & 0.87 & 1.45 & 2.25 \\
\hline Novelty & 1.69 & 1.19 & 1.15 & 2.24 \\
\hline Dependability & 1.57 & 0.78 & 1.21 & 1.93 \\
\hline Stimulation & 1.40 & 1.20 & 0.85 & 1.95 \\
\hline Efficiency & 1.39 & 0.76 & 1.04 & 1.74 \\
\hline
\end{tabular}

Comparing our results with a benchmark of data from other studies using the UEQ to evaluate interactive interfaces (Laugwitz et al., 2008); in general, our design was perceived by users as "Good" (Figure 7). Particularly, teachers agreed that BendableSound excels in attractiveness and novelty as both variables were scored as "Excellent". These results could be partially explained because teachers appreciated the interaction experience provided by the fabric and its multi-sensory stimuli. Our results also indicate teachers found BendableSound "easy to use" and are motivated in using it, as the variables of learnability, dependability, and stimulation were scored as "Good". This could be partially explained because teachers found the combination of open-ended activities and goal-oriented tasks to be appropriate to support their therapeutic practices.

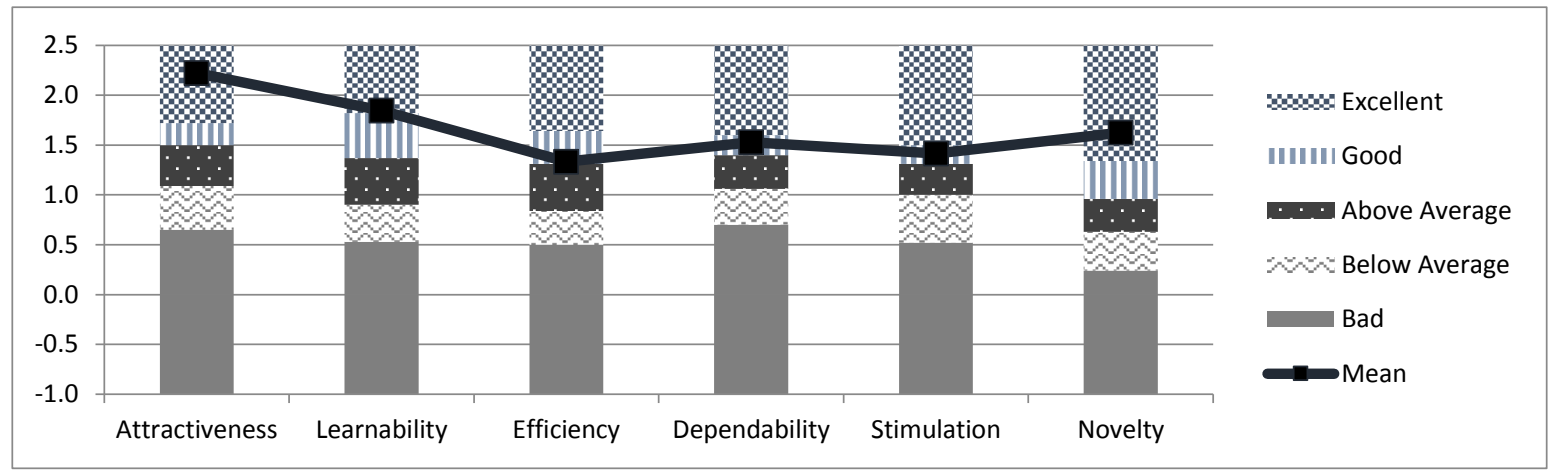

Figure 7. Results of the UEQ relative to the benchmarks

\subsubsection{Use and adoption}

The psychologist reported that participants found BendableSound "easy to use" by rapidly learning how to interact with the fabric and perceiving it as a useful tool.

"Performing [the activity] was really simple and smooth [referring to the use of BendableSound]. BendableSound facilitated children to play music ..." psychologist".

${ }^{9}$ All the quotes were transcribed by a non-native English speaker and the psychologist was non-native English speakers. 
Our results reflect that for around $80 \%$ of the time, in both conditions, our participants did not exhibit emotions (Table 5). From those who exhibited emotions, $71 \%{ }^{10}$ participants exhibited positive emotions using BendableSound and 58\% of participants exhibited positive emotions when playing with the Piano ( $\mathrm{p}=0.35$, Figure 8 ). For both conditions, close to $5 \%$ of the time participants exhibited a negative emotion $(\mathrm{p}=0.52)$. Our statistical analysis shows no significant differences between the two conditions when analyzing positive and negative emotions. But according to the psychologist's perception, when using BendableSound, participant's emotions were mostly positive and more intense and visible (Figure 8).

"Some participants [that could pronounce some words] said some expressions of astonishment like 'wow' or 'thanks'. [...] I think it was due to the multiple sensory stimuli available in the fabric, the game, the stars and everything available in the fabric. The fabric is what children like the most" psychologist

Table 5. Comparative of the positive and negative emotions participants exhibited using BendableSound (BS) and the Piano, on average. Note that the total exceeds $100 \%$ because participants could exhibit both positive and negative emotions on each condition

\begin{tabular}{|l|l|l|l|l|l|l|}
\cline { 2 - 7 } \multicolumn{1}{c|}{} & \multicolumn{2}{l|}{ Exhibited an emotion } & \multicolumn{2}{l|}{ Positive emotion } & \multicolumn{2}{l|}{ Negative emotion } \\
\cline { 2 - 7 } \multicolumn{1}{c|}{} & Piano & BS & Piano & BS & Piano & BS \\
\hline \% Participants & $75 \%$ & $83.33 \%$ & $58.33 \%$ & $70.83 \%$ & $33.33 \%$ & $20.83 \%$ \\
\hline Total time (sec) & 997 & 1471 & 814 & 1074 & 363 & 397 \\
\hline Avg. Time (sec) & 49 & 61 & 34 & 45 & 15 & 17 \\
\hline Avg. \% time & $16.34 \%$ & $20.43 \%$ & $11.31 \%$ & $14.92 \%$ & $5.03 \%$ & $5.51 \%$ \\
\hline
\end{tabular}
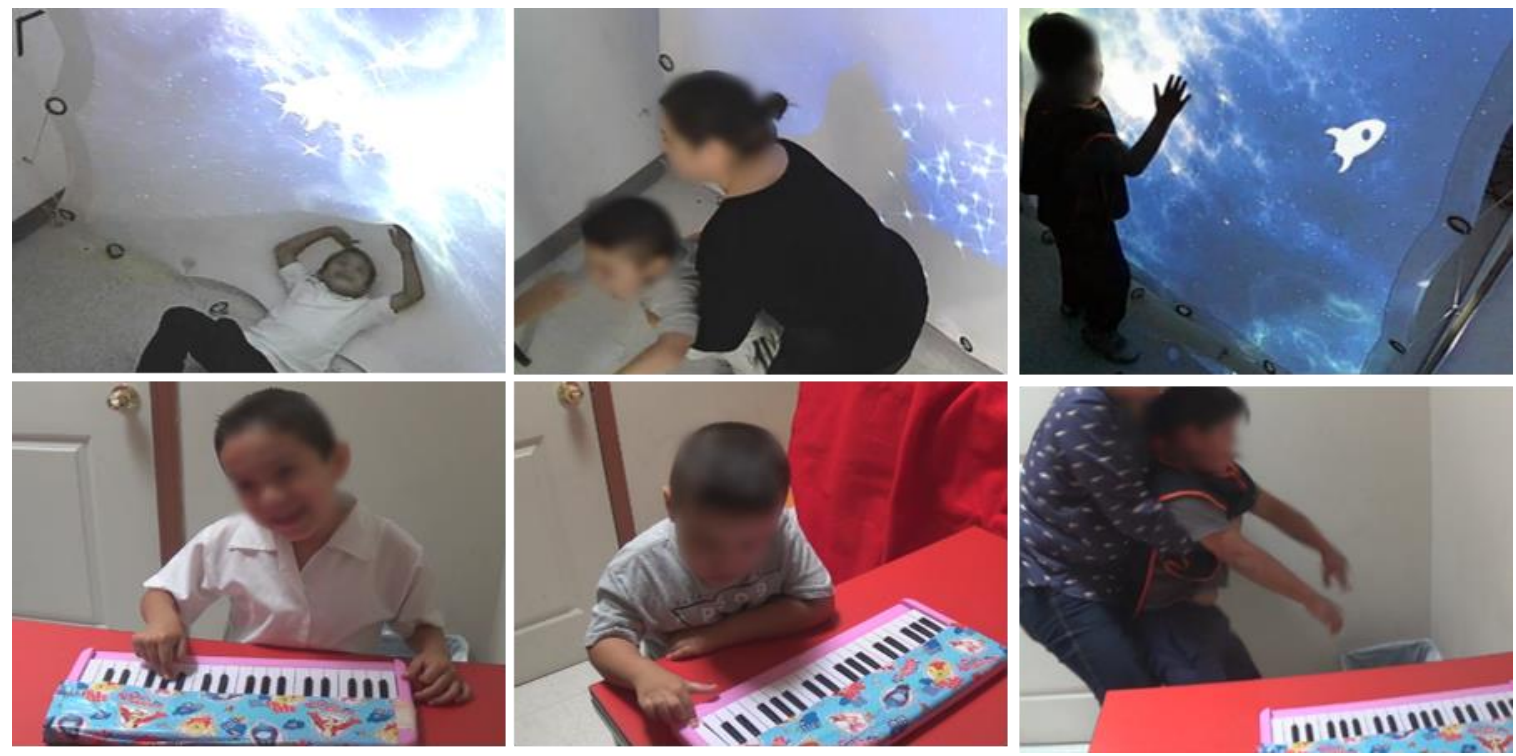

Figure 8. Participants in both conditions. Participant 3 laughing and enjoying the use of both BendableSound (left-up) and the keyboard piano (left- down). Participant 15 fighting with the therapist when using Bendable Sound (center-up) and being calmed and focused when using the keyboard piano (center-down). Participant 6 engaged and enjoying the use of BendableSound (right- up) but crying and refusing to use the keyboard piano (right-down)

When we queried the psychologist about the reasons for some participants exhibiting negative emotions when using BendableSound, she attributed such negative behaviors to the lack of tolerance of children with severe autism to disruptions to their routine and their fear of being exposed to new and novel experiences (American Psychiatric Association, 2013). Most negative emotions participants' exhibited when using the piano were related to being bored and frustrated (Figure 8).

${ }^{10}$ Percentages were calculated as a relation of the number of participants exhibiting the described behavior divided by the 24 children with autism who participated in the study. 
These results show that BendableSound offers an enjoyable experience and, for most participants, the multi-sensory experience worked. But, open questions remain concerning how BendableSound could adapt its stimuli according to the context of use (e.g., by reducing the stimuli for those children that need less stimulation). This will demand not only to personalize the visualizations and the sounds available in BendableSound but also to propose new methods for emotion recognition. We believe the body language and gestural interaction of the children using BendableSound could be used to infer their emotional state.

\subsubsection{The interaction experience}

We observed different interactions when participants used BendableSound. Participants enjoyed swiping and touching the fabric, either by using one finger, multiple fingers or their open hand (Figure 9). But they also used their whole body or specific body parts, like their heads or their back, to discover and explore movements they cannot do with traditional touch-based surfaces (Figure 9). Natural and novel interactions are important to help participants voluntarily and individually discover and explore new ways to interact with an interactive surface. The psychologist made several references to the importance of facilitating the interaction with the multisensory stimuli during therapies,

"The interaction [with BendableSound] is easy and enabled participants to interact with the fabric [...] [BendableSound] encouraged children to take on the initiative to uncover new and novel ways to interact with the music and with the fabric" Psychologist

The natural interaction and the combination of haptic, tactile, auditory, and visual sensations offered by BendableSound positively work together to increase children's curiosity and improve their overall experience during therapy sessions. Research has shown multisensory environments using haptic sensations may be better accepted by children with autism (Sitdhisanguan et al., 2012). Our results show it is important to use a variety of multisensory stimuli to provide a richer sensory stimulation for children with autism during therapies.
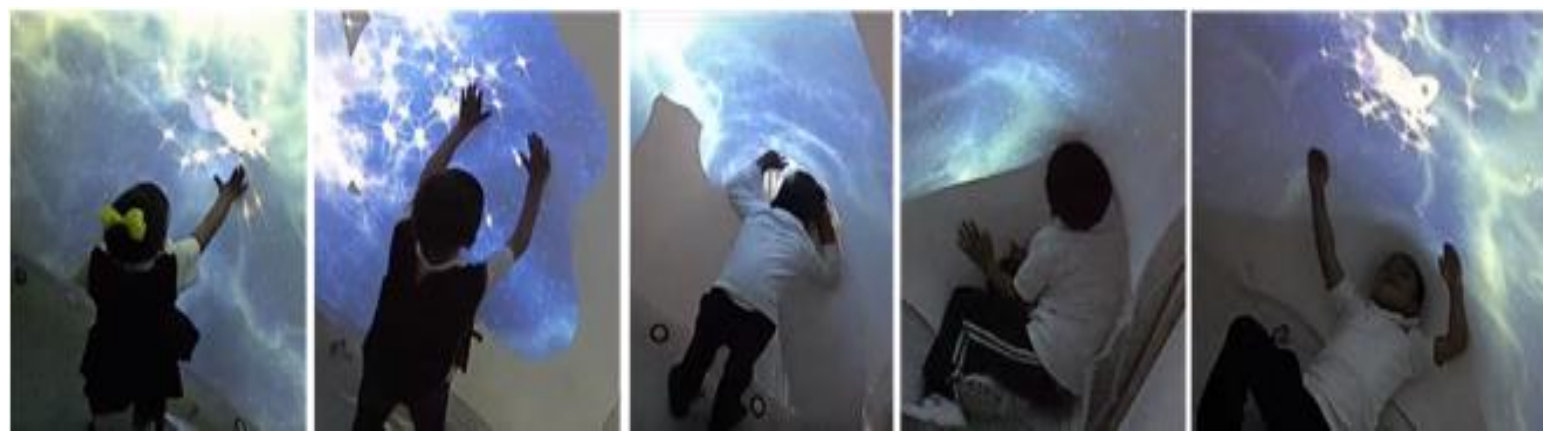

Figure 9. Participants with autism using different body parts to interact with BendableSound. From left to right, a girl using her hand to push the fabric; a boy pushing with both hands; a boy pushing with his head; a boy sitting to push the fabric with almost the whole body; a boy throwing in the floor watching and touching the fabric.

\subsection{Potential therapeutic benefits}

BendableSound helps children with autism to maintain their attention during therapy. Participants were able to practice different movements exploring the use of different parts of their body to interact with BendableSound, these results might indicate BendableSound could have therapeutic benefits regarding motor development and control of motor skills. 
International Journal of Human-Computer Studies: Special Issue on "Multisensory HumanComputer Interaction" 2016

\subsubsection{Attention and engagement}

$79 \%$ of the participants were paying more attention to the session when using BendableSound than with the piano and, participants were $7 \%$ more time focused on the therapy $(p=0.02)$. We found that BendableSound excelled the piano in improving the sustained attention of participants. Participants doubling the time they stay focused using BendableSound $(2.13$ more $)$ than with the keyboard piano (Table $6, p=0.003$ ). $45 \%$ of the participants spend more than 1 minute consecutively engaged in the therapy when interacting with BendableSound ( $25 \%$ of them spend more than 2 minutes). In contrast, only $16 \%$ of participants stayed focused when using the Piano (only $8 \%$ of them spend more than 2 minutes) (Figure 10).

Table 6. Comparative of the sustained attention that participants spend in both condition.

\begin{tabular}{|l|l|l|}
\cline { 2 - 3 } \multicolumn{1}{c|}{} & \multicolumn{2}{|l|}{ Sustained Attention } \\
\cline { 2 - 3 } \multicolumn{1}{c|}{} & Piano & BendableSound \\
\hline Avg. (sec.) & 40.14326 & 85.8654 \\
\hline Avg. of attention periods (\#) & 5.5 & 8.45 \\
\hline Children spend $>60$ sec. $(\#)$ & 4 & 11 \\
\hline
\end{tabular}

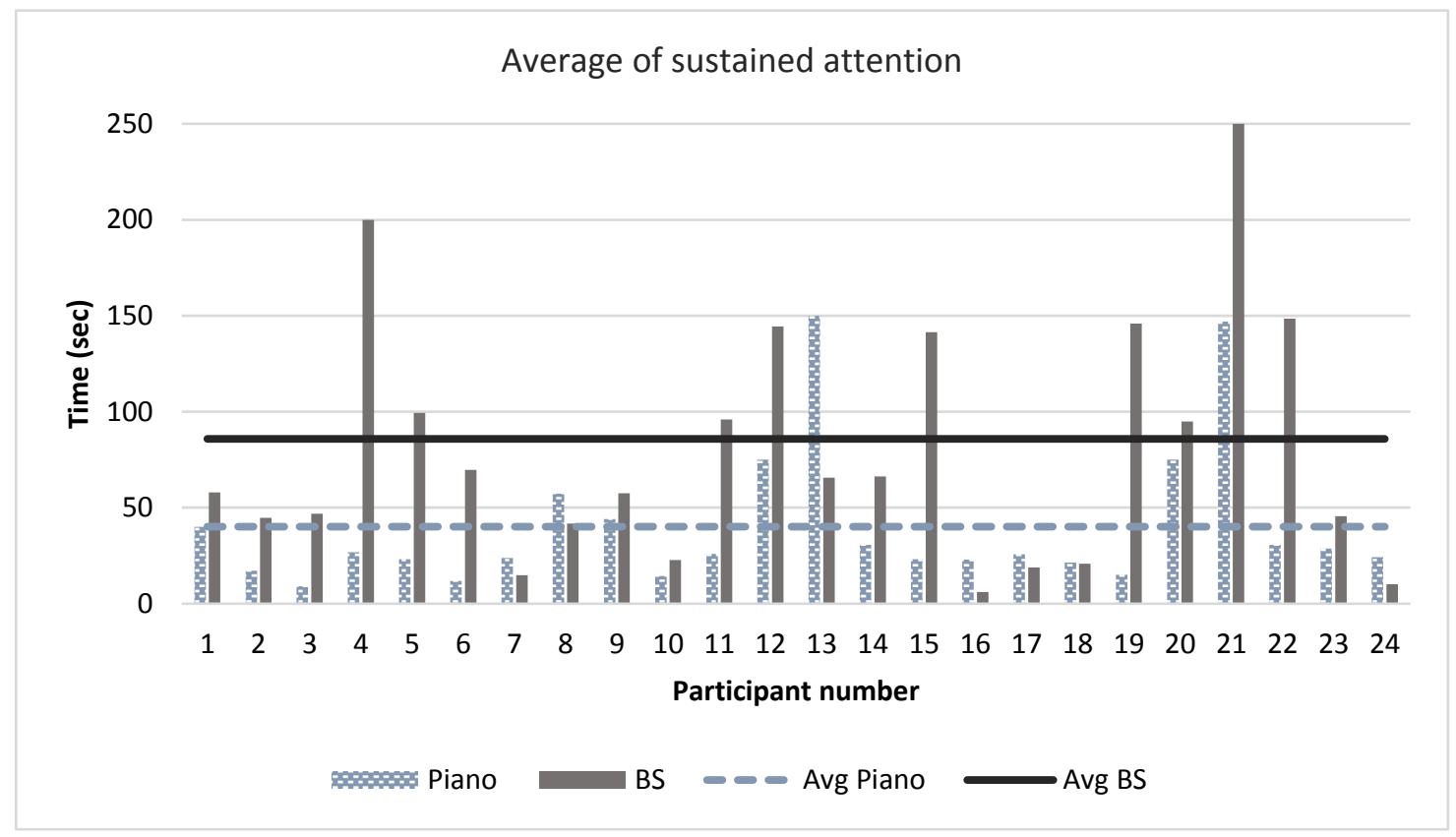

Figure 10. Average of time in seconds that participants spend focused on the therapy continuously using piano against using BendableSound (BS).

The psychologist attributes this improvement in participants' attention to the BendableSound's multisensory stimuli:

"I think [participants] like [BendableSound], they like to see the stars and hear the sounds. BendableSound is something new, they like to erase the black layer, they have the goal of fully illuminating the fabric. This represents a challenge for them ..." Psychologist.

"[With the piano, participants] were frustrated ... and I do not think their frustration was because of the keyboard or the piano itself; rather because the piano did not catch their attention like [BendableSound] does. As a consequence, they were most of the time distracted during the therapy" Psychologist 
Our results show BendableSound captures participants' attention in a more effective way than the piano. As a consequence, participants were more time exposed to sounds and music, increasing their chances to improve their engagement and adequately supporting a TIMP-NMT session. These results could indicate that BendableSound provides appropriate multi-sensory stimuli to maintain participants' attention during open-ended activities. Similar results in the literature show that a multisensory environment can improve engagement and attention of children with autism during therapies (Parés et al., 2005; Ringland et al., 2014). However, as the evaluation was conducted in a short period of time, open questions remain to study if these results are maintained in long-term and how participants' attention change after the novelty effect wears off.

\subsubsection{Potential motor development}

Although on average, participants performed 1.7 more gestures using BendableSound than with the piano, there was no significance difference $(p=0.33$, Table 7$) .99 \%$ of such gestures performed by participants involved the use of their hands, but $87 \%$ of participants tried to use their back, head or feet when interacting with BendableSound. Only $33 \%$ of the participants tried these gestures when using the Piano. Participants performed almost four times more movements using their back, head or feet with BendableSound than with the Piano $(\mathrm{p}=0.01)$. According to the psychologist, she believes that these novel movements could have a potential impact on gross motor development and strength control:

"I believe BendableSound [could have a potential impact] on gross motor skills, [children] have to push stronger, and sometimes they pushed the fabric in a softer way. Children also made figures on top of the fabric with their hands, these exercises could help them in the development of motor skills. I love this part!' Psychologist

Table 7. Comparative of the frequency of body parts that participants perform using BendableSound (BS) and the Piano. Note that we compare hands movement and we group foot, back, heat as using different body parts.

\begin{tabular}{|l|l|l|l|l|l|l|}
\cline { 2 - 7 } \multicolumn{1}{c|}{} & \multicolumn{2}{l|}{ Using Hands } & \multicolumn{2}{l|}{ Using different Body Part } & \multicolumn{2}{l|}{ Total } \\
\cline { 2 - 7 } \multicolumn{1}{c|}{} & Piano & BS & Piano & BS & Piano & BS \\
\hline Total & 761 & 803 & 19 & 75 & 780 & 878 \\
\hline Avg. & 31.708 & 33.458 & 0.79 & 3.125 & 32.5 & 36.58 \\
\hline SD & 15.81 & 14.20 & 1.18 & 3.77 & 16.04 & 16.31 \\
\hline
\end{tabular}

We also observe that participants varied their posture that could potentially help them to improve their balance and BendableSound may support the practicing of physical exercises that demand a change in their gait.

Overall, these results might indicate that BendableSound promotes the exploration of novel interaction gestures. According to the psychologist perception, children with severe autism may also gain a better understanding of movement concepts using BendableSound. This could potentially evolve into a deeper understanding of their movements and improvements in their sense of agency as other have pointed out with other populations (Bellan et al., 2017; Longo and Haggard, 2009). However, most motor therapeutic interventions for children with severe autism are highly structured filled with prompts and visual supports to guide the movements of users. Our design was instrumental in integrating both open-ended and goal-oriented tasks that could potentially encourage the repetition of these type of interactions. 
International Journal of Human-Computer Studies: Special Issue on "Multisensory HumanComputer Interaction" 2016

\section{Implications for designing multisensory deformable surfaces}

Reflecting on these results, we have identified a set of design recommendations for Ubicomp and HCI researchers interested in developing multisensory interactive surfaces to support therapeutic interventions for challenging populations.

\subsection{Providing adequate feedback of the haptic experience}

The use of multisensory stimuli to provide users with appropriate feedback when physically manipulating the fabric could enable a meaningful and a better haptic experience. But creating such "representation" of the haptic experience is not an easy task, especially when the design aims to support vulnerable populations with sensorimotor problems and sensory disorders. The way the user taps, touches or manipulates the surface should also modify the multisensory stimuli to give users a sense of control over their interactions and a complete experience of the effects of such interactions. This sense of agency has been suggested that it mainly arises from processes serving motor control (Moore and Fletcher, 2012), and facilitates the sensory processing and motor responses (Longo and Haggard, 2009).

In our case, for example, we decided to use a spandex fabric as a surface to take advantage of the haptic feedback provided by the fabric. The spandex fabric helped users to realize other potential interaction modalities with digital information; like understanding that digital artifacts can bend and "computer screens" could be soft and may be pushed. These interactions are most of the time impossible or very challenging to explore when using rigid surfaces. In BendableSound users can feel how much pressure they are using when touching the fabric even when they use different parts of their body.

In line with recent research in ubiquitous computing (Branje and Fels, 2014; Follmer et al., 2012; Giordano and Wanderley, 2013), we propose to take advantage of the haptic sensations provided by either using active "vibrotactile" or passive "textured" materials when designing multisensory surfaces. However, open questions remain to combine active and passive stimuli to improve the user experience and create novel haptic experiences.

\subsection{Balancing independence vs caregivers guidance}

Most of the interventions for people with disabilities are either behavioral or developmental (Ospina et al., 2008). Behavioral interventions are highly structured following discrete trial procedures heavily guided by therapists. For example, to improve motor performance, the same movement should be done repeatedly (Schmidt and Lee, 1988). In contrast, developmental interventions are more pragmatic enabling the patient to guide the therapy and demanding from therapists to follow children's interests (Ospina et al., 2008). Both paradigms provide share commonalities, such us, a systematic progression towards goals. The success of any approach will depend of the needs and preferences of each child, and the therapists' experience to balance both paradigms.

For example, BendableSound combines both open-ended and goal-oriented activities to enable the user to be independent but at the same time allowing the therapist to guide the user when appropriate. The open-ended activities available in BendableSound mimic improvisation techniques used during TIMP-NMT sessions including the discovery of the background where users can manipulate the stimuli according to their interests. In contrast, to promote repetition it is necessary to provide more guidance to patients. Repetition is necessary to improve movement trajectories and improve movement control (Büitefisch et al., 1995; Sterr et al., 2002). In this stage, the role of the stimuli 
guides children's activities; for example, in BendableSound the rocket position should invite the patient to use their left, right or both hands to catapult the rocket and collect the musical notes.

We recommend that multisensory surfaces must combine both open-ended and goal-oriented activities. And the multisensory stimuli must be arranged accordingly. However, there are open research questions around how to transition gradually from heavily guided interventions to interventions without prompts.

\subsection{Promoting natural and simple interactions}

One of the most popular ways to interact with computers is by using a mouse and a keyboard; both input devices, however, may inhibit our natural abilities of interaction (Malizia and Bellucci, 2012). Even though the mouse is a revolutionary device that changed how we use computers, it is not 'natural'. Users must learn how to control the mouse, and this could be challenging, especially for children, older adults, or people with disabilities. Natural user interfaces try to mimic how humans interact in the real world. (Malizia and Bellucci, 2012).

In our case, BendableSound, as an elastic multisensory surface, allows users to use gestures to vary the amount of pressure used when touching the surface (Müller et al., 2015) and explore novel ways of interaction. This gestural interaction model invites users to grasp, push, or bend the surface, which in turn lets them discover multisensory interaction experiences. Our interaction model has a dual functionality; it is both engaging and appropriate for motor development. So children could get the therapeutic benefits while having fun.

Using multisensory environments could provide a natural setting that allows a greater and more efficient learning practices (Shams and Seitz, 2008), even for children with autism (Parés et al., 2005; Ringland et al., 2014). Interactive multisensory surfaces are ideal to present immediate feedback to children with autism that can be introduced and regulated gently (Kientz et al., 2013), allowing children with autism to acquire successfully skills (Parés et al., 2005; Ringland et al., 2014). An interactive multisensory surface should incorporate a gameplay dynamic mimicking the type of rewards and prompts therapists gives to patients. Having an appropriate gameplay dynamic would help users to explore the surface, uncover new features, and solve challenges more directed to improve their skills.

\section{Conclusions and future work}

In this paper, we describe the co-design process we followed to develop the BendableSound prototype. Through both a formative and a deployment study we found out BendableSound is easy to use, useful and may have therapeutic benefits for both attention and motor development.

From a design standpoint, we learned that the use of novel haptic experiences combined with auditory and visual stimuli could help children with autism to stay focused in the therapy and allowed them to uncover novel ways of interaction. We learned the importance of using appropriate stimuli when designing elastic multisensory surfaces. Teachers suggested that we could include more challenges to allow children more rapidly master the physical exercises and encourage the use of BendableSound in long-term. For example, auditory stimuli may include timbre variations. The visual stimuli could include a wide range of themes such as a forest- or an ocean-based theme, and the haptic sensations could involve a broad range of textures. It will be interesting to explore the integration of other mechanisms from other techniques used in NMT. For example, mimicking activities from the Patterned Sensory Enhancement technique (Thaut and Hoemberg, 2014) could demand the use sonification techniques to be incorporated into elastic multisensory surfaces. 
From a technical standpoint of view, our results indicate psychologists perceived the installation of BendableSound in the clinic to be too complicated and not portable enough. Currently, we are working with industrial designers to uncover other potential materials and arrangement of our infrastructure to improve the set-up of BendableSound. Even though safety issues are a common problem when working with vulnerable populations and interactive technology, as others have reported (Vega-Barbas et al., 2015); we regret the lack of advances in technology that enable us to come up with a set up that is truly standalone, portable and easy to deploy. Overall, the development and deployment of elastic multisensory surfaces are heavily limited due to the lack of advances in the hardware currently being used for projection and the sensing hardware to detect where the user is touching.

Although the research reached our goals, we observed some unavoidable limitations. This research was conducted in only one school-clinic of children with autism. Therefore, to generalize the results for other populations, the study should be replicated with a much larger population. Additionally, children with autism only play once with BendableSound, then, the study should be replicated in long term to avoid the bias due to the "novelty effect". However, given that the objective of this study was not to generalize our findings but to get an overall impression of the potential of BendableSound in a particular use case, we feel that our results are valuable for researchers exploring the design space of elastic multisensory surfaces to support individuals with disabilities. As future work, we plan to conduct a longer deployment study to test the efficacy of using BendableSound as a therapeutic intervention to improve the self-regulation of the strength of children with autism, and investigate other clinical and educations benefits.

\section{Acknowledgments}

We thank all the participants enrolled in this study, and the researchers and the reviewers who provide helpful comments on previous versions of this document. This work was partially funded by the Microsoft Faculty Fellowship grant and the UCMexus grant for the fourth author. We also thank CONACYT for students' fellowships

\section{Author Biographies.}

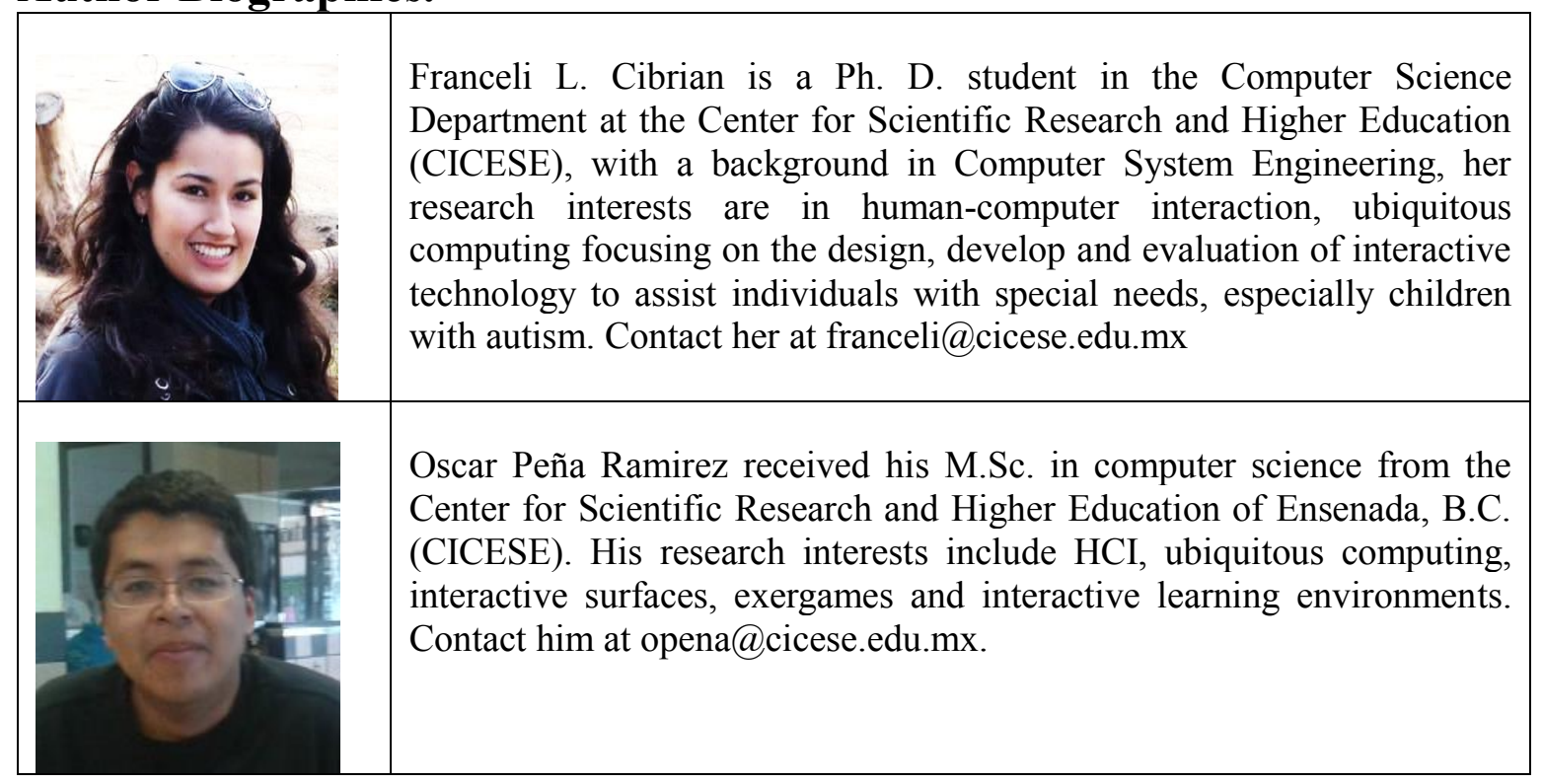




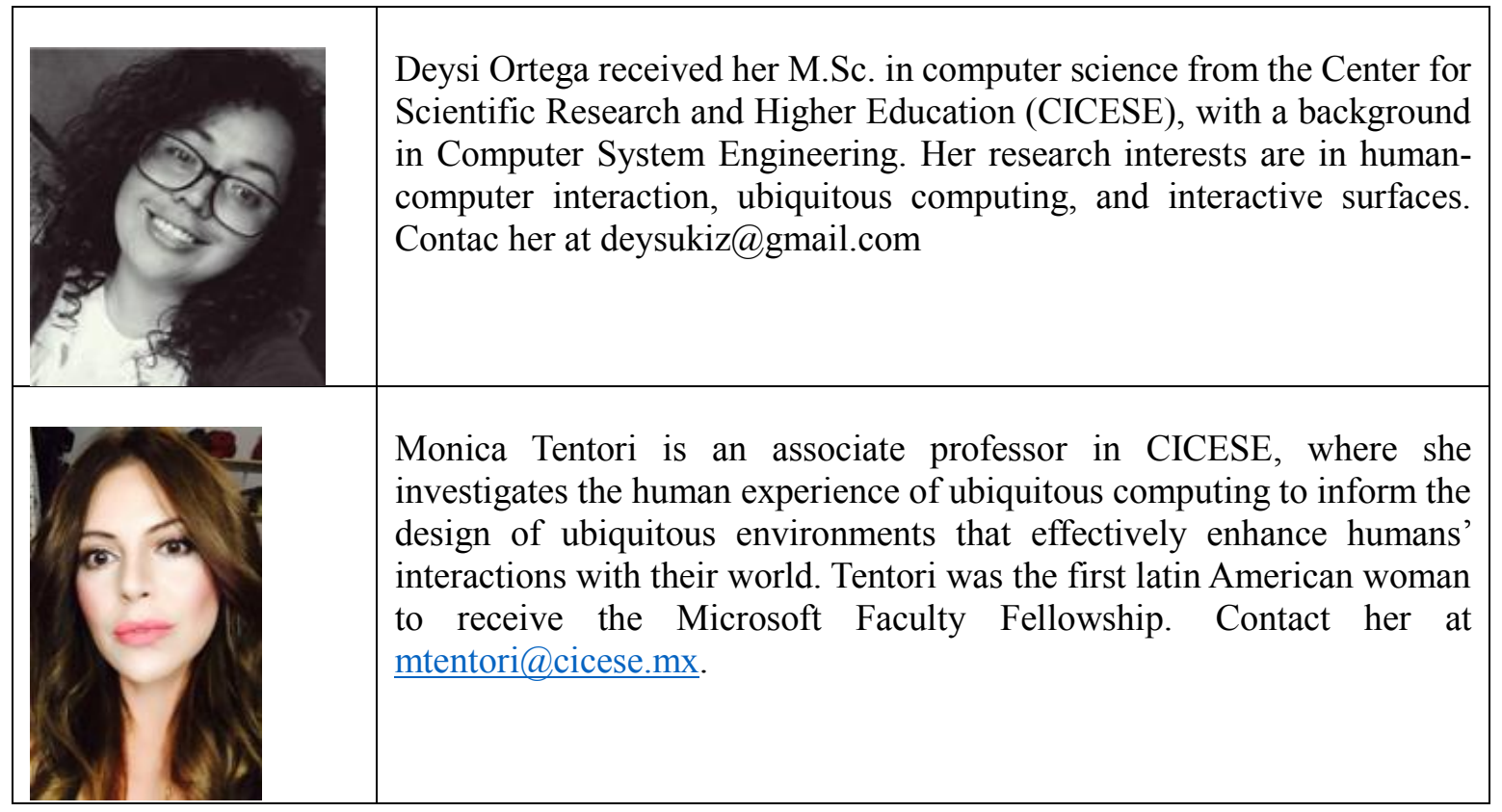

\section{References}

American Psychiatric Association, 2013. Diagnostic and Statistical Manual of Mental Disorders (DSM-5®), American Psychiatric Pub. doi:10.1176/appi.books.9780890425596.744053

Bangor, A., Kortum, P.T., Miller, J.T., 2008. An empirical evaluation of the system usability scale. Int. J. Hum. Comput. Interact. 24, 574-594. doi:10.1080/10447310802205776

Bellan, V., Ph, D., Wallwork, S.B., Hons, B.P., Gallace, A., Ph, D., Spence, C., Ph, D., Moseley, G.L., Sc, D., Ph, D., 2017. Integrating self-localization, proprioception, pain, and performance. J. Danc. Med. Sci. 21, 24-35. doi:10.12678/1089-313X.21.1.24

Berger, D.S., 2002. Music theapy, sensory integration and autistic child. Jessica Kingsley Publishers, London and New York. doi:10.1017/CBO9781107415324.004

Boulay, M., Benveniste, S., Boespflug, S., Jouvelot, P., Rigaud, A.S., 2011. A pilot usability study of MINWii, a music therapy game for demented patients. Technol. Heal. Care 19, 233-246. doi:10.3233/THC-2011-0628

Brakke, K., Fragaszy, D.M., Simpson, K., Hoy, E., Cummins-Sebree, S., 2007. The production of bimanual percussion in 12- to 24-month-old children. Infant Behav. Dev. 30, 2-15. doi:10.1016/j.infbeh.2005.08.001

Branje, C., Fels, D.I., 2014. Playing vibrotactile music: A comparison between the Vibrochord and a piano keyboard. Int. J. Hum. Comput. Stud. 72, 431-439. doi:10.1016/j.ijhcs.2014.01.003

Brooke, J., 2013. SUS : a retrospective. J. Usability Stud. 8, 29-40.

Büitefisch, C., Hummelsheim, H., Denzler, P., Mauritz, K.H., 1995. Repetitive training of isolated movements improves the outcome of motor rehabilitation of the centrally paretic hand. J. Neurol. Sci. 130, 59-68. doi:10.1016/0022-510X(95)00003-K

Burland, K., Magee, W., 2012. Developing identities using music technology in therapeutic settings. Psychol. Music 42, 177-189. doi:10.1177/0305735612463773

Cassinelli, A., Ishikawa, M., 2005. Khronos projector. ACM SIGGRAPH 2005 Emerg. Technol. SIGGRAPH '05 10. doi:10.1145/1187297.1187308

Cesarini, D., Calvaresi, D., Farnesi, C., Taddei, D., Frediani, S., Ungerechts, B.E., Hermann, T., 2016. MEDIATION: An eMbEddeD system for auditory feedback of hand-water InterAcTION while swimming. Procedia Eng. 147, 324-329. doi:10.1016/j.proeng.2016.06.301

Charmaz, K., 2006. Constructing grounded theory: a practical guide to through qualitative analysis. Sage Publications Ltd. 
Cibrian, F.L., Pena, O., Vazquez, V., Cardenas, C., Tentori, M., 2016a. Designing a deformable musical surface for children with autism, in: Proceedings of the 2016 ACM International Joint Conference on Pervasive and Ubiquitous Computing Adjunct - UbiComp '16. pp. 977-982. doi:10.1145/2968219.2968262

Cibrian, F.L., Weibel, N., Tentori, M., 2016b. Collective use of a fabric-based interactive surface to support early development in toddler classrooms, in: Proceedings of the 2016 ACM International Joint Conference on Pervasive and Ubiquitous Computing - UbiComp '16. pp. 328-339. doi:10.1145/2971648.2971695

Cuvo, A.J., May, M.E., Post, T.M., 2001. Effects of living room, Snoezelen room, and outdoor activities on stereotypic behavior and engagement by adults with profound mental retardation. Res. Dev. Disabil. 22, 183-204. doi:10.1016/S0891-4222(01)00067-1

Darrow, A.A., 2009. Adapting for students with autism. Gen. Music Today 22, 24-26. doi:10.1177/1048371308328384

Fagard, J., Wolff, P.H., 1991. The development of timing control and temporal organization in coordinated action: invariant relative timing, rhythms and coordination. Elsevier. doi:10.1017/CBO9781107415324.004

Follmer, S., Leithinger, D., Olwal, A., Cheng, N., Ishii, H., 2012. Jamming user interfaces: programmable particle stiffness and sensing for malleable and shape-changing devices, in: Proceedings of the 25th Annual ACM Symposium on User Interface Software and Technology - UIST'12. pp. 519-528. doi:10.1145/2380116.2380181

Fournier, K.A., Hass, C.J., Naik, S.K., Lodha, N., Cauraugh, J.H., 2010. Motor coordination in autism spectrum disorders: A synthesis and meta-analysis. J. Autism Dev. Disord. 40, 1227-1240. doi:10.1007/s10803-010-0981-3

Giordano, M., Wanderley, M.M., 2013. Perceptual and technological issues in the design of vibrotactile-augmented interfaces for music technology and media. Lect. Notes Comput. Sci. (including Subser. Lect. Notes Artif. Intell. Lect. Notes Bioinformatics) 7989 LNCS, 89-98. doi:10.1007/978-3-642-41068-0_10

Gorman, M., Lahav, A., Saltzman, E., Betke, M., 2007. A camera-based music-making tool for physical rehabilitation. Comput. Music J. 31, 39-53. doi:10.1162/comj.2007.31.2.39

Green, D., Charman, T., Pickles, A., Chandler, S., Loucas, T., Simonoff, E., Baird, G., 2009. Impairment in movement skills of children with autistic spectrum disorders. Dev. Med. Child Neurol. 51, 311-316. doi:10.1111/j.1469-8749.2008.03242.x

Grierson, M., Kiefer, C., 2013. NoiseBear: a malleable wireless controller designed in participation with disabled children. Proc. Int. Conf. New Interfaces Music. Expr. 413-416.

Grosshauser, T., Bläsing, B., Spieth, C., Hermann, T., 2012. Wearable sensor-based real-time sonification of motion and foot pressure in dance teaching and training. J. Audio Eng. Soc. 60, 580-589.

Grunder, T., Kammer, D., Brade, M., Groh, R., 2013. Towards a design space for elastic displays, in: CHI'13 Extended Abstracts on Human Factors in Computing Systems on - CHI EA '13. p. 4.

Hardy, M.W., Lagasse, a B., 2013. Rhythm, movement, and autism: using rhythmic rehabilitation research as a model for autism. Front. Integr. Neurosci. 7, 1-9. doi:10.3389/fnint.2013.00019

Hayes, G.R., Hirano, S., Marcu, G., Monibi, M., Nguyen, D.H., Yeganyan, M., 2010. Interactive visual supports for children with autism. Pers. Ubiquitous Comput. 14, 663-680. doi:10.1007/s00779-010-0294-8

Hobbs, D., Worthington-Eyre, B., 2008. The efficacy of combining augmented reality and music therapy with traditional teaching - Preliminary results. i-CREATe 2008 - Int. Conv. Rehabil. Eng. Assist. Technol. 2008 241-244.

Hopkins, I.M., Gower, M.W., Perez, T.A., Smith, D.S., Amthor, F.R., Casey Wimsatt, F., Biasini, F.J., 2011. Avatar assistant: Improving social skills in students with an asd through a computer-based intervention. J. Autism Dev. Disord. 41, 1543-1555. doi:10.1007/s10803-011-1179-z

Hourcade, J.P., Bullock-Rest, N.E., Hansen, T.E., 2012. Multitouch tablet applications and activities 
to enhance the social skills of children with autism spectrum disorders. Pers. Ubiquitous Comput. 16, 157-168. doi:10.1007/s00779-011-0383-3

Ichino, J., Pon, A., Sharlin, E., Eagle, D., Carpendale, S., 2014. Vuzik: the effect of large gesture interaction on children's creative musical expression, in: Proceedings of the 26th Australian Computer-Human Interaction Conference on Designing Futures: The Future of Design. ACM, pp. 240-249.

Jordà, S., Geiger, G., Alonso, M., Kaltenbrunner, M., 2007. The reacTable: exploring the synergy between live music performance and tabletop tangible interfaces, in: Proceedings of the 1st International Conference on Tangible and Embedded Interaction. ACM, New York, NY, USA, pp. 15-17. doi:10.1145/1226969.1226998

Keele, S.W., Ivry, R.I., Pokorny, R. a, 1987. Force control and its relation to timing. J. Mot. Behav. 19, 96-114. doi:10.1080/00222895.1987.10735402

Kientz, J.A., Goodwin, M.S., Hayes, G.R., Abowd, G.D., 2013. Interactive technologies for autism, Synthesis Lectures on Assistive, Rehabilitative, and Health-Preserving Technologies. Morgan \& Claypool Publishers.

LaGasse, A.B., Hardy, M.W., 2013. Considering rhythm for sensorimotor regulation in children with autism spectrum disorders. Music Ther. Perspect. 31, 67-77. doi:10.1093/mtp/31.1.67

Laugwitz, B., Held, T., Schrepp, M., 2008. Construction and evaluation of a user experience questionnaire. HCI Usability Educ. Work 63-76. doi:10.1007/978-3-540-89350-9_6

Longo, M.R., Haggard, P., 2009. Sense of agency primes manual motor responses. Perception 38, 6978. doi:10.1068/p6045

Lundy-Ekman, L., Ivry, R., Keele, S., Woollacott, M., 1991. Timing and force control deficits in clumsy children. J. Cogn. Neurosci. 3, 367--376.

Magee, W.L., 2006. Electronic technologies in clinical music therapy: A survey of practice and attitudes. Technol. Disabil. 18, 139-146.

Malizia, A., Bellucci, A., 2012. The artificiality of natural user interfaces. Commun. ACM 55, 36. doi:10.1145/2093548.2093563

Meldrum, D., Glennon, A., Herdman, S., Murray, D., McConn-Walsh, R., 2012. Virtual reality rehabilitation of balance: assessment of the usability of the Nintendo Wii(®) Fit Plus. Disabil. Rehabil. Assist. Technol. 7, 205-10. doi:10.3109/17483107.2011.616922

Mertel, K., 2014. Therapeutical Instrumental Music Performance (TIMP), in: Handbook of Neurologic Music Therapy. Oxford University Press, pp. 116-139.

Mintzberg, H., 1970. Structured observation as a method to study managerial work. J. Manag. Stud. 7, 87-104.

Moore, J.W., Fletcher, P.C., 2012. Sense of agency in health and disease: A review of cue integration approaches. Conscious. Cogn. 21, 59-68. doi:10.1016/j.concog.2011.08.010

Müller, M., Gründer, T., Groh, R., 2015. Data exploration on elastic displays using physical metaphors, in: xCoAx 2015: Proceedings of the Third Conferenc on Computation, Communication, Aesthetics and X. pp. 111-124.

Nawaz, A., Helbostad, J.L., Chiari, L., Chesani, F., Cattelani, L., 2015. User Experience (UX) of the fall risk assessment tool (FRAT-up). 2015 IEEE 28th Int. Symp. Comput. Med. Syst. 19-22. doi:10.1109/CBMS.2015.63

Oliveros, P., Miller, L., Heyen, J., Siddall, G., Hazard, S., 2011. A musical improvisation interface for people with severe physical disabilities. Music Med. 3, 172-181. doi: $10.1177 / 1943862111411924$

Ortega, D., Cibrian, F.L., Tentori, M., 2015. BendableSound: a fabric-based interactive surface to promote free play in children with autism, in: In Proceedings of the 16th International ACM SIGACCESS Conference on Computers \& Accessibility. ACM, pp. 315-316. doi: $10.1145 / 2700648.2811355$

Ospina, M.B., Seida, J.K., Clark, B., Karkhaneh, M., Hartling, L., Tjosvold, L., Vandermeer, B., Smith, V., 2008. Behavioural and developmental interventions for autism spectrum disorder: A 
International Journal of Human-Computer Studies: Special Issue on "Multisensory HumanComputer Interaction" 2016

clinical systematic review. PLoS One 3. doi:10.1371/journal.pone.0003755

Parés, N., Soler, M., Sanjurjo, À., Carreras, A., Durany, J., Ferrer, J., Freixa, P., Gómez, D., Kruglanski, O., Parés, R., Ribas, J.I., 2005. Promotion of creative activity in children with severe autism through visuals in an interactive multisensory environment. Proceeding 2005 Conf. Interact. Des. Child. - IDC '05 110-116. doi:10.1145/1109540.1109555

Piochon, C., Kloth, A.D., Grasselli, G., Titley, H.K., Nakayama, H., Hashimoto, K., Wan, V., Simmons, D.H., Eissa, T., Nakatani, J., Cherskov, A., Miyazaki, T., Watanabe, M., Takumi, T., Kano, M., Wang, S.S.-H., Hansel, C., 2014. Cerebellar plasticity and motor learning deficits in a copy-number variation mouse model of autism. Nat. Commun. 5, 5586. doi:10.1038/ncomms6586

Putnam, C., Chong, L., 2008. Software and technologies designed for people with autism: what do users want?, in: Proceedings of the 10th International ACM SIGACCESS Conference on Computers and Accessibility. ACM, pp. 3-10. doi:10.1145/1414471.1414475

Rauschenberger, M., Schrepp, M., Perez-Cota, M., Olschner, S., Thomaschewski, J., 2013. Efficient measurement of the user experience of interactive products. How to use the user experience questionnaire (UEQ). Example: spanish language version. Int. J. Interact. Multimed. Artif. Intell. 2, 39-45. doi:10.9781/ijimai.2013.215

Repp, B.H., Su, Y.-H., 2013. Sensorimotor synchronization: a review of recent research (2006-2012). Psychon. Bull. Rev. 20, 403-52. doi:10.3758/s13423-012-0371-2

Ringland, K.E., Zalapa, R., Neal, M., Escobedo, L., Tentori, M., Hayes, G.R., 2014. SensoryPaint: a multimodal sensory intervention for children with neurodevelopmental disorders, in: Proceedings of the 2014 ACM International Joint Conference on Pervasive and Ubiquitous Computing. pp. 873--884.

Rogers, K., Amrei, R., Weing, M., Gugenheimer, J., Bastian, K., Klepsch, M., Schaub, F., Rukzio, E., Seufert, T., Weber, M., 2014. P . I . A . N . O .: Faster piano learning with interactive projection, in: Proceedings of the Ninth ACM International Conference on Interactive Tabletops and Surfaces. ACM, pp. 149-158. doi:10.1145/2669485.2669514

Rosati, G., Roda, A., Avanzini, F., Masiero, S., 2013. On the role of auditory feedback in roboticassisted movement training after stroke. Comput. Intell. Neurosci. 2013.

Sahoo, D.R., Hornbæk, K., Subramanian, S., 2016. TableHop: an actuated fabric display using transparent electrodes. Proc. 2016 CHI Conf. Hum. Factors Comput. Syst. 3767-3780. doi: $10.1145 / 2858036.2858544$

Salgado-Montejo, A., Marmolejo-Ramos, F., Alvarado, J.A., Arboleda, J.C., Suarez, D.R., Spence, C., 2016. Drawing sounds: representing tones and chords spatially. Exp. Brain Res. 1-14. doi: $10.1007 / \mathrm{s} 00221-016-4747-9$

Santoso, H.B., Isal, R.Y.K., Basaruddin, T., Sadira, L., Schrepp, M., 2014. Research-in-progress: User experience evaluation of student centered e-learning environment for computer science program. 2014 3rd Int. Conf. User Sci. Eng. 52-55. doi:10.1109/IUSER.2014.7002676

Schaaf, R.C., Benevides, T., Mailloux, Z., Faller, P., Hunt, J., van Hooydonk, E., Freeman, R., Leiby, B., Sendecki, J., Kelly, D., 2014. An intervention for sensory difficulties in children with autism: a randomized trial. J. Autism Dev. Disord. 44, 1493-506. doi:10.1007/s10803-013-1983-8

Schaffert, N., Mattes, K., 2015. Interactive sonification in rowing: Acoustic feedback for on-water training. IEEE Multimed. 22, 58-67. doi:10.1109/MMUL.2015.9

Schmidt, R.A., Lee, T., 1988. Motor control and learning: A behavioral emphasis, 2nd ed. ed. Champaign, IL: Human kinetics.

Schmidt, R.A., Wrisberg, C.A., 2008. Motor learning and performance: A situation-based learning approach, 4th ed. ed. Human kinetics.

Schonauer, C., Pintaric, T., Kaufmann, H., Jansen-Kosterink, S., Vollenbroek-Hutten, M., 2011. Chronic pain rehabilitation with a serious game using multimodal input. 2011 Int. Conf. Virtual Rehabil. ICVR 2011. doi:10.1109/ICVR.2011.5971855

Shams, L., Seitz, A.R., 2008. Benefits of multisensory learning. Trends Cogn. Sci. 12, 411-417. 
International Journal of Human-Computer Studies: Special Issue on "Multisensory HumanComputer Interaction" 2016

doi:10.1016/j.tics.2008.07.006

Sigrist, R., Rauter, G., Marchal-Crespo, L., Riener, R., Wolf, P., 2014. Sonification and haptic feedback in addition to visual feedback enhances complex motor task learning. Exp. Brain Res. 233, 909-925. doi:10.1007/s00221-014-4167-7

Singh, A., Piana, S., Pollarolo, D., Volpe, G., Varni, G., Tajadura-Jiménez, A., Williams, A.C., Camurri, A., Bianchi-Berthouze, N., 2015. Go-with-the-Flow: tracking, analysis and sonification of movement and breathing to build confidence in activity despite chronic pain. Human-Computer Interact. 24, 1-49. doi:10.1080/07370024.2015.1085310

Sitdhisanguan, K., Chotikakamthorn, N., Dechaboon, A., Out, P., 2012. Using tangible user interfaces in computer-based training systems for low-functioning autistic children. Pers. Ubiquitous Comput. 16, 143-155. doi:10.1007/s00779-011-0382-4

Spence, C., 2011. Crossmodal correspondences: A tutorial review 971-995. doi:10.3758/s13414-0100073-7

Srinivasan, S.M., Bhat, A.N., 2013. A review of "music and movement" therapies for children with autism: embodied interventions for multisystem development. Front. Integr. Neurosci. 7, 1-15. doi:10.3389/fnint.2013.00022

Staples, K.L., Reid, G., 2010. Fundamental movement skills and autism spectrum disorders. J. Autism Dev. Disord. 40, 209-17. doi:10.1007/s10803-009-0854-9

Sterr, a, Freivogel, S., Voss, a, 2002. Exploring a repetitive training regime for upper limb hemiparesis in an in-patient setting: a report on three case studies. Brain Inj. 16, 1093-107. doi:10.1080/02699050210155267

Tang, S.T., McCorkle, R., 2002. Use of family proxies in quality of life research for cancer patients at the end of life: a literature review. Cancer Invest. 20, 1086-1104. doi:10.1081/CNV120005928

Tartaro, A., Cassell, J., 2008. Playing with virtual peers: bootstrapping contingent discourse in children with autism, in: Proceedings of the 8th International Conference on International Conference for the Learning Sciences - Volume 2. pp. 382-389.

Thaut, M.H., Hoemberg, V., 2014. Handbook of Neurologic Music Therapy. Oxford University Press.

Torres, E.B., Donnellan, A.M., 2015. Autism: The Movement Perspective. Frontiers in Integrative Neuroscience. doi:10.3389/978-2-88919-509-1

Troiano, G.M., Pedersen, E.W., Hornbæk, K., 2014. User-defined gestures for elastic, deformable displays. Proc. 2014 Int. Work. Conf. Adv. Vis. Interfaces - AVI '14 1-8. doi: $10.1145 / 2598153.2598184$

Troiano, G.M., Pedersen, E.W., Hornbæk, K., Copenhagen, D.-, 2015. Deformable interfaces for performing music, in: Proceedings of the 33rd Annual ACM Conference on Human Factors in Computing Systems. ACM, New York, NY, USA, pp. 377-386. doi:10.1145/2702123.2702492

Vega-Barbas, M., Pau, I., Ferreira, J., Lebis, E., Seoane, F., 2015. Utilizing smart textiles-enabled sensorized toy and playful interactions for assessment of psychomotor development on children. J. Sensors 2015, 1-9. doi:10.1155/2015/898047

Villafuerte, L., Markova, M., Jorda, S., 2012. Acquisition of social abilities through musical tangible user interface: Children with autism spectrum condition and the Reactable, in: CHI'12 Extended Abstracts on Human Factors in Computing Systems. ACM, New York, NY, USA, pp. 745-760. doi:10.1145/2212776.2212847

Visi, F., Schramm, R., Miranda, E., 2014. Gesture in performance with traditional musical instruments and electronics - Use of embodied music cognition and multimodal motion capture to design gestural mapping strategies. Proc. Int. Work. Mov. Comput. 100-105. doi: $10.1145 / 2617995.2618013$

Wendell, J.B., Shelley, W., Karen, H., 2004. Rapid contextual design A How-To Guide to Key Techniques for User-Centered Design. Elsevier. doi:10.1145/1066322.1066325

Wigram Tony, Pedersen Inge Nygaard, B.L.O., 2011. A Comprehensive Guide to Music Therapy Theory, Clinical Practice, Research and Training. 
International Journal of Human-Computer Studies: Special Issue on "Multisensory HumanComputer Interaction" 2016

Xiao, X., Puentes, P., Ackermann, E., Ishii, H., 2016. Andantino: Teaching children piano with projected animated characters, in: ACM SIGCHI Conference on Interaction Design and Children 2016. pp. 37-45.

Yun, K., Song, J., Youn, K., Cho, S., Bang, H., 2013. ElaScreenn: Exploring multi-dimensional data using elastic screen. CHI '13 Ext. Abstr. Hum. Factors Comput. Syst. - CHI EA '13 1311. doi: $10.1145 / 2468356.2468590$ 